Prepared for the U.S. Department of Energy

under Contract DE-AC05-76RL01830

\title{
Final Report for DHS FY10 Uranium Methodology Project Analyses of PTRM10 1.1, 1.2, 3.1, and 3.2
}

KL Noyes

SL Petersen

SJ Garofoli

KB Wagnon

November 2010
MM Huff

SM Schulte

JI Friese 


\title{
DISCLAIMER
}

This report was prepared as an account of work sponsored by an agency of the United States Government. Neither the United States Government nor any agency thereof, nor Battelle Memorial Institute, nor any of their employees, makes any warranty, express or implied, or assumes any legal liability or responsibility for the accuracy, completeness, or usefulness of any information, apparatus, product, or process disclosed, or represents that its use would not infringe privately owned rights. Reference herein to any specific commercial product, process, or service by trade name, trademark, manufacturer, or otherwise does not necessarily constitute or imply its endorsement, recommendation, or favoring by the United States Government or any agency thereof, or Battelle Memorial Institute. The views and opinions of authors expressed herein do not necessarily state or reflect those of the United States Government or any agency thereof.

\author{
PACIFIC NORTHWEST NATIONAL LABORATORY \\ operated by \\ BATTELLE \\ for the \\ UNITED STATES DEPARTMENT OF ENERGY \\ under Contract DE-AC05-76RL01830
}

Printed in the United States of America
Available to DOE and DOE contractors from the Office of Scientific and Technical Information,
P.O. Box 62, Oak Ridge, TN 37831-0062;
ph: (865) 576-8401
fax: $(865) 576-5728$
email: reports@adonis.osti.gov

\footnotetext{
Available to the public from the National Technical Information Service, U.S. Department of Commerce, 5285 Port Royal Rd., Springfield, VA 22161 ph: (800) 553-6847 fax: $(703) 605-6900$ email: orders@ntis.fedworld.gov online ordering: http://www.ntis.gov/ordering.htm
}

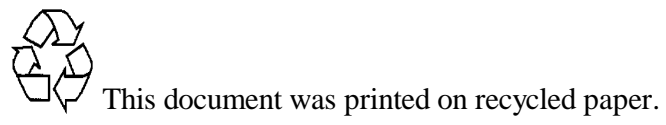




\section{Final Report for DHS FY10 Uranium Methodology Project \\ Analyses of PTRM10 1.1, 1.2, 3.1, and 3.2}

KL Noyes, SL Petersen, SJ Garofoli, KB Wagnon, MM Huff, SM Schulte, and JI Friese $11 / 30 / 2010$

This report contains a description of activities performed in support of the FY10 Uranium Methodologies Project at PNNL, as well as the final data from the analyses. 


\section{Measurement Scheme}

The samples were received in lab 329/131, then transferred to lab 329/121 for aliquotting and distribution for analyses. Alpha aliquots were chemically separated and electrodeposited in lab 329/121, with alpha counting taking place in 329/16A. Thermal ionization mass spectrometry (TIMS) aliquots were transferred to lab 320/113 for TIMS source preparation under radiological control; no chemical separations were performed on TIMS aliquots. In both labs, each set of replicate samples were accompanied by one chemical (laboratory) process blank for quality assurance purposes.

All ${ }^{232} \mathrm{U} /{ }^{234} \mathrm{U}$ ratios are measured via alpha energy analysis (AEA). The following ratios were measured by TIMS: ${ }^{233} \mathrm{U} /{ }^{235} \mathrm{U},{ }^{234} \mathrm{U} /{ }^{235} \mathrm{U},{ }^{236} \mathrm{U} /{ }^{235} \mathrm{U}$, and ${ }^{238} \mathrm{U} /{ }^{235} \mathrm{U}$, where ${ }^{235} \mathrm{U}$ was the dominant isotope; analogous measurements were made in cases where ${ }^{238} \mathrm{U}$ was the dominant isotope. $A{ }^{232} \mathrm{U} /{ }^{235} \mathrm{U}$ and ${ }^{232} \mathrm{U} /{ }^{238} \mathrm{U}$ ratios are calculated using a combination of the AEA and TIMS data.

\section{Methods Used}

The methods used in these analyses are the same methods that are normally used on samples and are described in the SOPs in the following section. Not all of the methods in the SOPs section were used; this report does not cover PTRM10-2. Chemical separations were not necessary for the TIMS samples, but were required for the AEA samples.

\section{SOPs Used}

1. 320-A-13: U Determinations by TIMS for NTNFC Samples

2. 320-113-3: Sample Dissolution, Preliminary Analysis, Splitting, Spiking, and Equilibration for U and Pu Determinations

3. 320-113-2: Anion Exchange Separation and Purification of $U$ and Pu for Samples Containing ppm Levels of Iron

4. 320-A-11: TIMS Source Preparation for Purified U and Pu Fractions of Samples

5. Alpha Calibrations [not yet a formalized procedure]

6. 329-AEA-1: Alpha Spectroscopy Sample Analysis

7. 329-ED-USTUR-1: 329-Electrodeposition of Radiological Samples via USTUR Method

8. 329-ED-EICM-1: 329-Electrodeposition of Radiological Samples via EICROM Method

9. 329-AEAPREP-01: Alpha Spectroscopy Sample Preparation

10. RC-UTEVA-TRU: Chemical Separations for Analysis of Actinides

11. 329-NC-1: Operations Protocol for Radiochemical Analytical Work Performed in 329 Building, Labs 121, 122, 126 and 127 


\section{Additional Planning/Processes}

None.

\section{Calculation of Uncertainties}

Uncertainties for individual measurements are propagated errors based on counting statistics, detector dark noise, and background noise (at mass 243 for TIMS). TIMS data is also corrected for mass bias based on the analysis of many natural uranium standards (CRM-129a) analyzed in a manner as similar as possible to the samples. Reported uncertainties are GUM compliant.

For the TIMS data, the single measurand values were calculated as an average of all replicates (10-11 measurements, depending on the sample). The GUM uncertainties were generated using the following general equation:

$$
\text { combined }_{\text {avgMP }}=\left(\left(\mathrm{X}_{\mathrm{MPDay} 1} * \delta_{\mathrm{MPBCFDay} 1}+\mathrm{X}_{\mathrm{MPDay} 2} * \delta_{\mathrm{MPBCFDay} 2}\right) / 2\right) * \delta_{\text {Reproducibility }} * \delta_{\text {Repeatability }}
$$

Where combined $\mathrm{avgMP}_{\text {is }}$ the combined average over both days for a ratio-ed minor mass $(\mathrm{M})$ to a primary mass $(P), X_{M P D a y 1}$ is the average ratio for the sample for the M/P ratio on day $1, X_{M P D a y 2}$ is the average ratio for the M/P ratio of the sample on day2, $\delta_{\text {MPBCFDay } 1}$ is the delta factor for the M/P ratio bias correction on day $1, \delta_{\mathrm{MPBCFDay} 2}$ is the delta factor for the M/P ratio bias correction on day $2, \delta_{\text {Reproducability }}$ is the delta factor for day-to-day reproducibility of the ${ }^{235} \mathrm{U} /{ }^{238} \mathrm{U}$ ratio of a certified isotopic standard, and $\delta_{\text {Repeatability }}$ is the delta factor characterizing the intra-day repeatability of the ${ }^{235} \mathrm{U} /{ }^{238} \mathrm{U}$ ratio of a certified isotopic standard.

The delta factors are entered as $1 \pm$ uncertainty at the 1-sigma level. The bias correction delta factor uncertainties are calculated as $(P-M) / 3 *\left(R S D_{B C F 58}\right)$, where $P$ is the primary mass, $M$ is the minor mass, and $\mathrm{RSD}_{\mathrm{BCF} 58}$ is the relative standard deviation of the bias correction factor for the ${ }^{235} \mathrm{U} /{ }^{238} \mathrm{U}$ ratio based on the 5-6 certified isotopic standards run at the same time as the PTRM10 sample they are associated with. The repeatability uncertainty is the relative standard deviation of ${ }^{235} \mathrm{U} /{ }^{238} \mathrm{U}$ ratio of the six certified isotopic standards run at the same time as the sample they are associated with. The reproducibility uncertainty is the relative standard deviation of the ${ }^{235} \mathrm{U} /{ }^{238} \mathrm{U}$ ratio of the 48 certified isotopic standards run throughout the PTRM10 analytical process.

\section{Number of Analysts Performing Tasks for this Project}

1. Sample Aliquoting: 2 analysts

2. Chemical Separations for AEA: 1 analyst

3. Electrodepositions for AEA: 1 analyst

4. Alpha Counting for AEA: 2 analysts

5. TIMS Source Loading for TIMS Samples: 1 analyst 
6. TIMS Source Loading for TIMS QA/QC Samples: 2 analysts

7. TIMS Source Processing: 2 analysts

8. TIMS Instrument Operation: 1 analyst

9. Alpha Data Analysis: 2 analysts

10. TIMS Data Analysis: 2 analysts

\section{Calibrations, Reference Materials, and Quality Control Samples}

The settings used for each mass on the TIMS instrument are calibrated quarterly with DAC check standards. Certified reference materials are run with each set of samples analyzed on the TIMS instrument as well (CRM-129a for uranium samples). Each set is co-processed with a chemical process blank as the quality control sample.

\section{Review Process for Analytical Data}

All data, both TIMS and alpha, undergoes a two-person review before it is ready for reporting. This review process entails checking for typos, ensuring that the correct subset of raw data has been used (for alpha, that the peak is appropriately defined; for TIMS, that invalid data points are excluded), and that calibrations/mass biases are appropriately incorporated.

\section{Deviations from, Additions to, or Exclusions from Analysis Methods}

None. 


\section{Appendix A: Measurement Data}

\section{U Methodology Isotopic Data Reporting}

\begin{tabular}{|c|c|c|c|}
\hline Facility Name: & PNNL & Division: & NSD \\
\hline Report Author: & KLN & Date: & $11 / 29 / 2010$ \\
\hline Analysis Method 1: & TIMS & Analyst 1: & \\
\hline Analysis Method 2: & AEA & Analyst 2: & \\
\hline Analysis Method 3: & & Analyst 3: & \\
\hline
\end{tabular}

\begin{tabular}{|c|c|c|c|c|c|c|c|}
\hline \multirow{2}{*}{ Sample ID } & \multirow{2}{*}{$\begin{array}{l}\text { Replicate } \\
\text { Number }\end{array}$} & Isotopic Ratio & Isotopic Ratio & Isotopic Ratio & Isotopic Ratio & Isotopic Ratio & \multirow{2}{*}{$\begin{array}{c}\text { Analysis } \\
\text { Date }\end{array}$} \\
\hline & & \begin{tabular}{|l|}
$232 U / 235 U$ \\
\end{tabular} & $233 \mathrm{U} / 235 \mathrm{U}$ & $234 \mathrm{U} / 235 \mathrm{U}$ & $236 \mathrm{U} / 235 \mathrm{U}$ & $238 \mathrm{U} / 235 \mathrm{U}$ & \\
\hline \multirow{15}{*}{ PTRM10-1.1 } & $86471 \mathrm{~A}$ & $7.83881 \mathrm{E}-10$ & $2.19408 \mathrm{E}-06$ & 0.009730798 & 0.015163155 & 0.55054734 & \multirow{7}{*}{$8 / 21 / 2010$} \\
\hline & $86471 \mathrm{~B}$ & $8.00 \mathrm{E}-10$ & $2.00018 \mathrm{E}-06$ & 0.009761917 & 0.015175165 & 0.551461606 & \\
\hline & $86471 \mathrm{C}$ & $8.05 \mathrm{E}-10$ & 2.34994E-06 & 0.009772958 & 0.015176105 & 0.551208952 & \\
\hline & $86471 \mathrm{D}$ & $7.98 \mathrm{E}-10$ & 2.19279E-06 & 0.009764111 & 0.015148191 & 0.551211033 & \\
\hline & \multicolumn{2}{|l|}{$86471 \mathrm{E}$} & $2.00503 E-06$ & 0.009766408 & 0.015171618 & 0.551091784 & \\
\hline & Average & 7.96585E-10 & 2.1484E-06 & 0.009759238 & 0.015166847 & 0.551104143 & \\
\hline & Std Uncert & 4.45189E-12 & 6.60298E-08 & 7.34653E-06 & 5.19299E-06 & 0.000151719 & \\
\hline & $86475 \mathrm{~A}$ & $8.02363 \mathrm{E}-10$ & $2.42268 \mathrm{E}-06$ & 0.009722974 & 0.015109038 & 0.550243287 & \multirow{8}{*}{$8 / 30 / 2010$} \\
\hline & $86475 B$ & $8.05548 \mathrm{E}-10$ & 2.40439E-06 & 0.009706158 & 0.015141825 & 0.549914578 & \\
\hline & $86475 \mathrm{C}$ & $8.00298 \mathrm{E}-10$ & $2.1744 \mathrm{E}-06$ & 0.009732986 & 0.015133193 & 0.550278577 & \\
\hline & $86475 \mathrm{D}$ & $8.10573 \mathrm{E}-10$ & $2.43275 \mathrm{E}-06$ & 0.009705294 & 0.015152122 & 0.551545784 & \\
\hline & $86475 \mathrm{E}$ & & $2.78279 \mathrm{E}-06$ & 0.009709785 & 0.015120466 & 0.549911968 & \\
\hline & $86475 \mathrm{~F}$ & & 2.06784E-06 & 0.009720329 & 0.015129225 & 0.549732549 & \\
\hline & Average & 8.04696E-10 & $2.38081 E-06$ & 0.009716254 & 0.015130978 & 0.550271124 & \\
\hline & Std Uncert & $2.23695 \mathrm{E}-12$ & 1.01184E-07 & 4.49301E-06 & $6.2305 \mathrm{E}-06$ & 0.000269058 & \\
\hline \multicolumn{2}{|c|}{ Combined Average } & 8.01E-10 & 2.27E-06 & 0.009738 & 0.015149 & 0.5507 & \\
\hline \multicolumn{2}{|c|}{ Expanded Uncertainty $\left(U=k \cdot u_{c}\right)$} & & $6.00 \mathrm{E}-08$ & 4.70E-05 & $7.30 \mathrm{E}-05$ & $2.90 \mathrm{E}-03$ & \\
\hline \multirow{2}{*}{ Sample ID } & \multirow{2}{*}{$\begin{array}{c}\text { Replicate } \\
\text { Number }\end{array}$} & Isotopic Ratio & Isotopic Ratio & Isotopic Ratio & Isotopic Ratio & Isotopic Ratio & \multirow{2}{*}{$\begin{array}{c}\text { Analysis } \\
\text { Date }\end{array}$} \\
\hline & & $232 U / 238 U$ & $233 \mathrm{U} / 238 \mathrm{U}$ & $234 \mathrm{U} / 238 \mathrm{U}$ & $235 \mathrm{U} / 238 \mathrm{U}$ & $236 \mathrm{U} / 238 \mathrm{U}$ & \\
\hline \multirow{14}{*}{ PTRM10-1.2 } & 86472A & $9.55518 \mathrm{E}-12$ & --- & 0.000411302 & 0.047457123 & 0.000290002 & \multirow{7}{*}{$8 / 6 / 2010$} \\
\hline & 86472B & $9.20876 \mathrm{E}-12$ & --- & 0.000408035 & 0.047397343 & 0.000290326 & \\
\hline & $86472 \mathrm{C}$ & $8.93176 \mathrm{E}-12$ & --- & 0.000408923 & 0.04735173 & 0.000289918 & \\
\hline & 86472D & & --- & 0.000411729 & 0.047529634 & 0.000291453 & \\
\hline & \multicolumn{2}{|l|}{$86472 E$} & --- & 0.000401937 & 0.047133707 & 0.000289821 & \\
\hline & Average & $9.2319 \mathrm{E}-12$ & \#DIV/0! & 0.000408385 & 0.047373907 & 0.000290304 & \\
\hline & Std Uncert & $1.80336 \mathrm{E}-13$ & \#DIV/0! & 1.75614E-06 & 6.70495E-05 & $2.99436 \mathrm{E}-07$ & \\
\hline & 86476A & & --- & 0.000403867 & 0.047363245 & 0.000287692 & \multirow{7}{*}{$8 / 13 / 2010$} \\
\hline & 86476B & & --- & 0.000406773 & 0.047461333 & 0.000289585 & \\
\hline & $86476 \mathrm{C}$ & & --- & 0.000409239 & 0.047385994 & 0.000289216 & \\
\hline & 86476D & & --- & 0.000407413 & 0.047402334 & 0.000290479 & \\
\hline & $86476 \mathrm{E}$ & & --- & 0.000405985 & 0.047264853 & 0.00028843 & \\
\hline & Average & \#DIV/0! & \#DIV/0! & 0.000406655 & 0.047375552 & 0.000289081 & \\
\hline & Std Uncert & \#DIV/0! & \#DIV/0! & $8.79888 \mathrm{E}-07$ & 3.20862E-05 & 4.78468E-07 & \\
\hline \multicolumn{2}{|c|}{ Combined Average } & & --- & 4.08E-04 & 0.04737 & $2.90 \mathrm{E}-04$ & \\
\hline \multicolumn{2}{|c|}{ Expanded Uncertainty $\left(U=k \cdot u_{c}\right)$} & & --- & $2.40 \mathrm{E}-06$ & $2.40 \mathrm{E}-04$ & $1.40 \mathrm{E}-06$ & \\
\hline
\end{tabular}




\section{U Methodology Isotopic Data Reporting}

\begin{tabular}{|c|c|c|c|}
\hline Facility Name: & PNNL & Division: & NSD \\
\hline Report Author: & KLN & Date: & $11 / 29 / 2010$ \\
\hline Analysis Method 1: & TIMS & Analyst 1: & \\
\hline Analysis Method 2: & AEA & Analyst 2: & \\
\hline Analysis Method 3: & & Analyst 3: & \\
\hline
\end{tabular}

\begin{tabular}{|c|c|c|c|c|c|c|c|}
\hline \multirow{2}{*}{ Sample ID } & \multirow{2}{*}{$\begin{array}{c}\text { Replicate } \\
\text { Number }\end{array}$} & Isotopic Ratio & Isotopic Ratio & Isotopic Ratio & Isotopic Ratio & Isotopic Ratio & \multirow{2}{*}{$\begin{array}{c}\text { Analysis } \\
\text { Date }\end{array}$} \\
\hline & & $232 \mathrm{U} / 238 \mathrm{U}$ & $233 \mathrm{U} / 238 \mathrm{U}$ & $234 \mathrm{U} / 238 \mathrm{U}$ & $235 \mathrm{U} / 238 \mathrm{U}$ & $236 \mathrm{U} / 238 \mathrm{U}$ & \\
\hline \multirow{15}{*}{ PTRM10-3.1 } & $86473 \mathrm{~A}$ & $1.98334 \mathrm{E}-11$ & -- & 0.000494615 & 0.05282504 & 0.000158349 & \multirow{7}{*}{$8 / 23 / 2010$} \\
\hline & $86473 B$ & $1.98 \mathrm{E}-11$ & --- & 0.000491909 & 0.05260334 & 0.00015898 & \\
\hline & $86473 C$ & $1.91 \mathrm{E}-11$ & --- & 0.000488544 & 0.052736766 & 0.000159703 & \\
\hline & $86473 \mathrm{D}$ & $1.92 \mathrm{E}-11$ & --- & 0.000494491 & 0.052799979 & 0.00015796 & \\
\hline & $86473 \mathrm{E}$ & & --- & 0.000490652 & 0.052759713 & 0.000159031 & \\
\hline & Average & 1.94812E-11 & \#DIV/0! & 0.000492042 & 0.052744967 & 0.000158805 & \\
\hline & Std Uncert & 1.98254E-13 & \#DIV/0! & $1.15753 \mathrm{E}-06$ & 3.85882E-05 & 3.00738E-07 & \\
\hline & \begin{tabular}{|l|}
$86477 A$ \\
\end{tabular} & & --- & 0.000493173 & 0.05300798 & 0.000159901 & \multirow{8}{*}{$8 / 31 / 2010$} \\
\hline & $86477 \mathrm{~B}$ & & --- & 0.000498534 & 0.052921012 & 0.000158215 & \\
\hline & $86477 \mathrm{C}$ & & --- & 0.000494448 & 0.052977792 & 0.000159461 & \\
\hline & 86477D & & --- & 0.000490501 & 0.052873863 & 0.000158569 & \\
\hline & $86477 \mathrm{E}$ & & --- & 0.00049569 & 0.053020497 & 0.000161082 & \\
\hline & $86477 \mathrm{~F}$ & & --- & 0.000495331 & 0.053022601 & 0.000160177 & \\
\hline & Average & \#DIV/0! & \#DIV/0! & 0.000494613 & 0.052970624 & 0.000159568 & \\
\hline & Std Uncert & \#DIV/0! & \#DIV/0! & $1.09629 \mathrm{E}-06$ & 2.48057E-05 & 4.32663E-07 & \\
\hline \multicolumn{2}{|c|}{ Combined Average } & & -- & 4.93E-04 & 0.05286 & 1.59E-04 & \\
\hline \multicolumn{2}{|c|}{ Expanded Uncertainty $\left(\mathrm{U}=\mathrm{k} \cdot \mathrm{u}_{\mathrm{c}}\right)$} & & $-\cdots$ & 2.60E-06 & $2.60 \mathrm{E}-04$ & 8.20E-07 & \\
\hline \multirow{2}{*}{ Sample ID } & \multirow{2}{*}{$\begin{array}{c}\text { Replicate } \\
\text { Number }\end{array}$} & Isotopic Ratio & Isotopic Ratio & Isotopic Ratio & Isotopic Ratio & Isotopic Ratio & \multirow{2}{*}{$\begin{array}{c}\text { Analysis } \\
\text { Date }\end{array}$} \\
\hline & & $232 U / 238 U$ & $233 \mathrm{U} / 238 \mathrm{U}$ & $234 \mathrm{U} / 238 \mathrm{U}$ & $235 \mathrm{U} / 238 \mathrm{U}$ & $236 \mathrm{U} / 238 \mathrm{U}$ & \\
\hline \multirow{15}{*}{ PTRM10-3.2 } & 86474A & $2.35245 \mathrm{E}-11$ & -- & 0.000502686 & 0.05303912 & 0.000168261 & \multirow{7}{*}{$8 / 9 / 2010$} \\
\hline & 86474B & $2.25466 \mathrm{E}-11$ & $--\cdot$ & 0.000498089 & 0.052965388 & 0.000168949 & \\
\hline & $86474 \mathrm{C}$ & 2.2929E-11 & --- & 0.000502844 & 0.052944809 & 0.000168515 & \\
\hline & 86474D & 2.27449E-11 & --- & 0.00049931 & 0.05285607 & 0.000168925 & \\
\hline & 86474E & & $--\cdot$ & 0.000503356 & 0.052681033 & 0.000168201 & \\
\hline & Average & 2.29363E-11 & \#DIV/0! & 0.000501257 & 0.052897284 & 0.00016857 & \\
\hline & Std Uncert & 2.11043E-13 & \#DIV/0! & 1.06761E-06 & 6.14318E-05 & 1.58703E-07 & \\
\hline & 86478A & & --- & 0.000499813 & 0.053077291 & 0.000165994 & \multirow{8}{*}{$8 / 16 / 2010$} \\
\hline & 86478B & & --- & 0.00050244 & 0.053174329 & 0.000166197 & \\
\hline & $86478 \mathrm{C}$ & & --- & 0.000496826 & 0.053036653 & 0.000169246 & \\
\hline & 86478D & & --- & 0.000503313 & 0.05301602 & 0.000167283 & \\
\hline & $86478 \mathrm{E}$ & & $--\cdot$ & 0.000503971 & 0.053055935 & 0.000168242 & \\
\hline & $86478 \mathrm{~F}$ & & $--\cdot$ & 0.000507268 & 0.053152491 & 0.000168306 & \\
\hline & Average & \#DIV/0! & \#DIV/0! & 0.000502272 & 0.053085453 & 0.000167545 & \\
\hline & Std Uncert & \#DIVI0! & \#DIVI0! & 1.46813E-06 & 2.61619E-05 & 5.24426E-07 & \\
\hline \multicolumn{2}{|c|}{ Combined Average } & & --- & 5.02E-04 & 0.05299 & 1.68E-04 & \\
\hline \multicolumn{2}{|c|}{ Expanded Uncertainty $\left(U=k \cdot u_{c}\right)$} & & --- & 3.00E-06 & 2.80E-04 & 8.90E-07 & \\
\hline
\end{tabular}




\section{Appendix B: Uncertainty Budgets}

Exhibit B.1: Uncertainty Budgets from PTRM10-1.1.

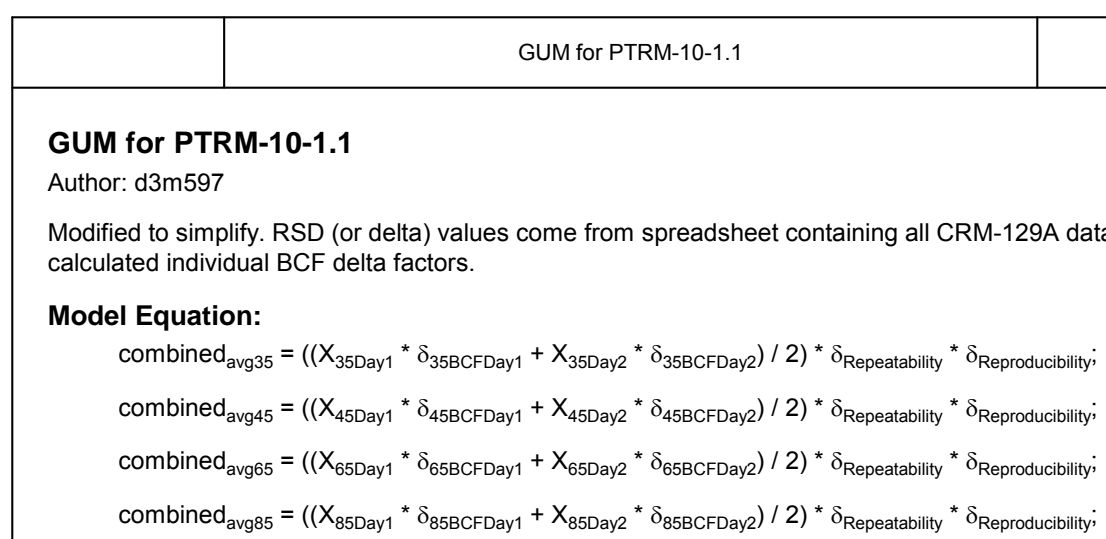

List of Quantities:

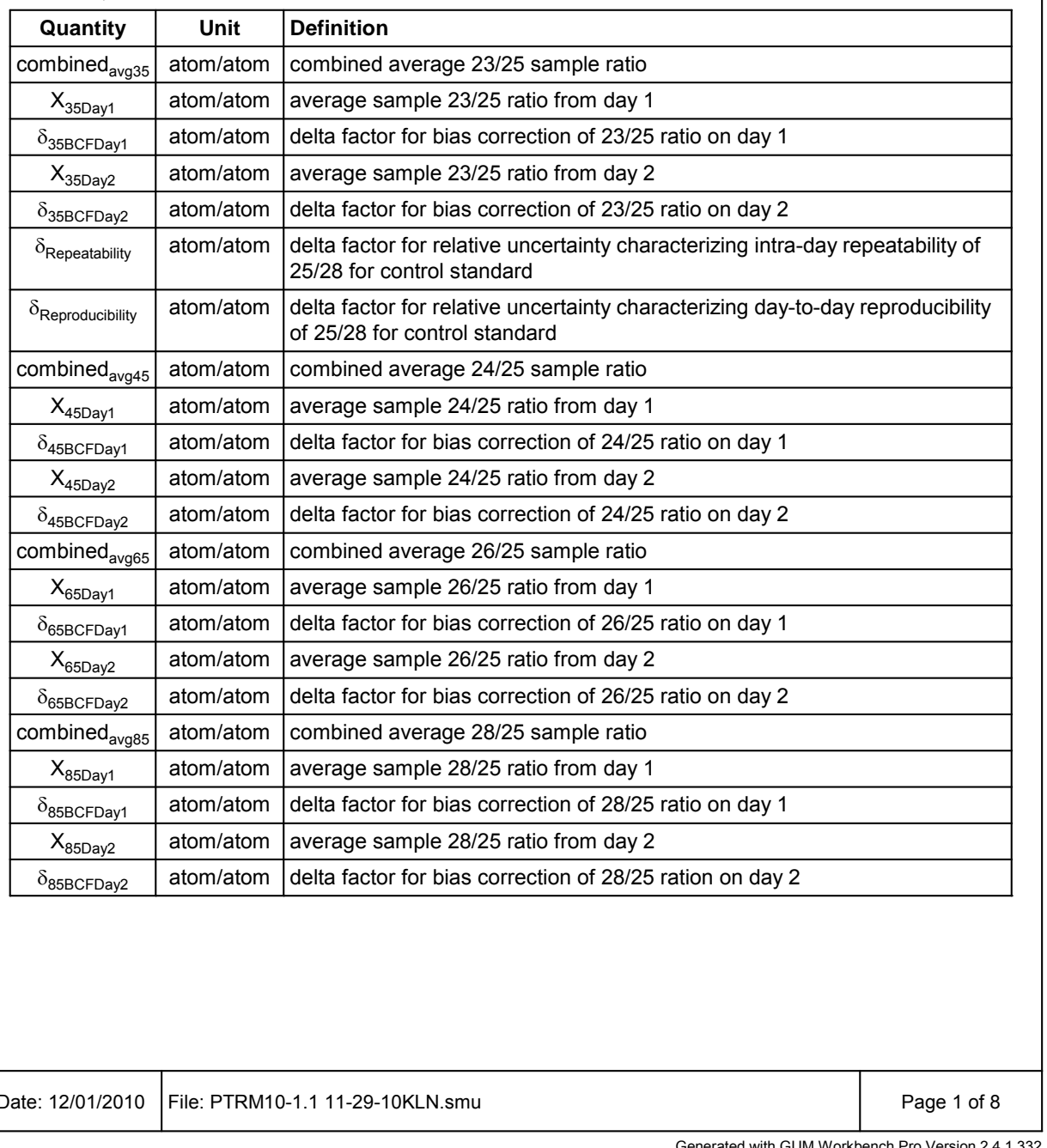


PNNL-20047

\begin{tabular}{|c|c|c|c|}
\hline & \multicolumn{2}{|r|}{ GUM for PTRM-10-1.1 } & \\
\hline \multirow[t]{8}{*}{$\mathrm{X}_{35 \mathrm{Day} 1}:$} & \multicolumn{2}{|c|}{$\begin{array}{l}\text { Type A } \\
\text { Method of observation: Direct } \\
\text { Number of observations: } 5\end{array}$} & \\
\hline & No. & Observation & \\
\hline & 1 & $2.1941 \cdot 10^{-6}$ atom/atom & \\
\hline & 2 & $\begin{array}{l}0.00000200018 \\
\text { atom/atom }\end{array}$ & \\
\hline & 3 & $\begin{array}{l}0.00000234994 \\
\text { atom/atom }\end{array}$ & \\
\hline & 4 & $\begin{array}{l}0.00000219279 \\
\text { atom/atom }\end{array}$ & \\
\hline & 5 & $\begin{array}{l}0.000002005029 \\
\text { atom/atom }\end{array}$ & \\
\hline & \multicolumn{2}{|c|}{$\begin{array}{l}\text { Arithmetic Mean: } 2.1484 \cdot 10^{-6} \text { atom/atom } \\
\text { Pooled Standard Deviation: } 0.000000066030 \text { atom/atom } \\
\text { Pooled Degrees of Freedom: } 49 \\
\text { Standard Uncertainty: } 33.7 \cdot 10^{-9} \text { atom/atom }\end{array}$} & \\
\hline$\delta_{35 B C F D a y 1}:$ & \multicolumn{2}{|c|}{$\begin{array}{l}\text { Type B normal distribution } \\
\text { Value: } 1 \text { atom/atom } \\
\text { Expanded Uncertainty: } 0.0014305 \text { atom/atom } \\
\text { Coverage Factor: } 1\end{array}$} & \\
\hline \multirow[t]{8}{*}{$\mathrm{X}_{35 \mathrm{Day2}}:$} & \multicolumn{2}{|c|}{$\begin{array}{l}\text { Type A } \\
\text { Method of observation: Direct } \\
\text { Number of observations: } 6\end{array}$} & \\
\hline & No. & Observation & \\
\hline & 1 & $\begin{array}{l}0.00000242268 \\
\text { atom/atom }\end{array}$ & \\
\hline & 2 & $\begin{array}{l}0.00000240439 \\
\text { atom/atom }\end{array}$ & \\
\hline & 3 & $\begin{array}{l}0.00000217440 \\
\text { atom/atom }\end{array}$ & \\
\hline & 4 & $\begin{array}{l}0.00000243275 \\
\text { atom/atom }\end{array}$ & \\
\hline & 5 & $\begin{array}{l}0.00000278279 \\
\text { atom/atom }\end{array}$ & \\
\hline & 6 & $\begin{array}{l}0.00000206784 \\
\text { atom/atom }\end{array}$ & \\
\hline & \multicolumn{2}{|c|}{$\begin{array}{l}\text { Arithmetic Mean: } 2.3808 \cdot 10^{-6} \text { atom/atom } \\
\text { Pooled Standard Deviation: } 0.000000097368 \text { atom/atom } \\
\text { Pooled Degrees of Freedom: } 49 \\
\text { Standard Uncertainty: } 48.8 \cdot 10^{-9} \text { atom/atom }\end{array}$} & \\
\hline$\delta_{35 B C F D a y 2}:$ & \multicolumn{2}{|c|}{$\begin{array}{l}\text { Type B normal distribution } \\
\text { Value: } 1 \text { atom/atom } \\
\text { Expanded Uncertainty: } 0.00049908 \text { atom/atom } \\
\text { Coverage Factor: } 1\end{array}$} & \\
\hline Date: $12 / 01 / 2010$ & \multicolumn{2}{|c|}{ File: PTRM10-1.1 11-29-10KLN.smu } & Page 2 of 8 \\
\hline
\end{tabular}

Generated with GUM Workbench Pro Version 2.4.1.332 


\begin{tabular}{|c|c|c|c|}
\hline & \multicolumn{2}{|r|}{ GUM for PTRM-10-1.1 } & \\
\hline$\delta_{\text {Repeatability }}:$ & \multicolumn{2}{|c|}{$\begin{array}{l}\text { Type B normal distribution } \\
\text { Value: } 1 \text { atom/atom } \\
\text { Expanded Uncertainty: } 0.0011178 \text { atom/atom } \\
\text { Coverage Factor: } 1\end{array}$} & \\
\hline$\delta_{\text {Reproducibility }}:$ & \multicolumn{2}{|c|}{$\begin{array}{l}\text { Type B normal distribution } \\
\text { Value: } 1 \text { atom/atom } \\
\text { Expanded Uncertainty: } 0.0021029 \text { atom/atom } \\
\text { Coverage Factor: } 1\end{array}$} & \\
\hline \multirow[t]{8}{*}{$\mathrm{X}_{45 \text { Day } 1}:$} & \multicolumn{2}{|c|}{$\begin{array}{l}\text { Type A } \\
\text { Method of observation: Direct } \\
\text { Number of observations: } 5\end{array}$} & \\
\hline & No. & Observation & \\
\hline & 1 & 0.009731 atom/atom & \\
\hline & 2 & 0.009762 atom/atom & \\
\hline & 3 & 0.009773 atom/atom & \\
\hline & 4 & 0.009764 atom/atom & \\
\hline & 5 & 0.009766 atom/atom & \\
\hline & \multicolumn{2}{|c|}{$\begin{array}{l}\text { Arithmetic Mean: } 9.75920 \cdot 10^{-3} \text { atom/atom } \\
\text { Pooled Standard Deviation: } 0.0000073465 \text { atom/atom } \\
\text { Pooled Degrees of Freedom: } 49 \\
\text { Standard Uncertainty: } 3.74 \cdot 10^{-6} \text { atom/atom }\end{array}$} & \\
\hline$\delta_{45 B C F D a y 1}:$ & \multicolumn{2}{|c|}{$\begin{array}{l}\text { Type B normal distribution } \\
\text { Value: } 1 \text { atom/atom } \\
\text { Expanded Uncertainty: } 0.00071524 \text { atom/atom } \\
\text { Coverage Factor: } 1\end{array}$} & \\
\hline \multirow[t]{8}{*}{$\mathrm{X}_{45 \mathrm{Day2} 2}:$} & \multicolumn{2}{|c|}{$\begin{array}{l}\text { Type A } \\
\text { Method of observation: Direct } \\
\text { Number of observations: } 6\end{array}$} & \\
\hline & No. & Observation & \\
\hline & 1 & 0.009723 atom/atom & \\
\hline & 2 & 0.009706 atom/atom & \\
\hline & 3 & 0.009733 atom/atom & \\
\hline & 4 & 0.009705 atom/atom & \\
\hline & 5 & 0.009710 atom/atom & \\
\hline & 6 & 0.009720 atom/atom & \\
\hline$\delta_{45 B C F D a y 2}:$ & $\begin{array}{l}\text { Arithmetic M } \\
\text { Pooled Stan } \\
\text { Pooled Degr } \\
\text { Standard Un } \\
\\
\text { Type B norm } \\
\text { Value: } 1 \text { ato } \\
\text { Expanded U } \\
\text { Coverage Fa }\end{array}$ & $\begin{array}{l}\text { Type B normal distribution } \\
\text { Value: } 1 \text { atom/atom } \\
\text { Expanded Uncertainty: } 0.00024954 \text { atom/atom } \\
\text { Coverage Factor: } 1\end{array}$ & \\
\hline Date: $12 / 01 / 2010$ & \multicolumn{2}{|c|}{ File: PTRM10-1.1 11-29-10KLN.smu } & Page 3 of 8 \\
\hline
\end{tabular}


PNNL-20047

\begin{tabular}{|c|c|c|c|}
\hline & \multicolumn{2}{|r|}{ GUM for PTRM-10-1.1 } & \\
\hline \multirow[t]{8}{*}{$\mathrm{X}_{65 \text { Day } 1}:$} & \multicolumn{2}{|c|}{$\begin{array}{l}\text { Type A } \\
\text { Method of observation: Direct } \\
\text { Number of observations: } 5\end{array}$} & \\
\hline & No. & Observation & \\
\hline & 1 & 0.015163 atom/atom & \\
\hline & 2 & 0.015175 atom/atom & \\
\hline & 3 & 0.015176 atom/atom & \\
\hline & 4 & 0.015148 atom/atom & \\
\hline & 5 & 0.015172 atom/atom & \\
\hline & \multicolumn{2}{|c|}{$\begin{array}{l}\text { Arithmetic Mean: } 0.01516680 \text { atom/atom } \\
\text { Pooled Standard Deviation: } 0.0000051930 \text { atom/atom } \\
\text { Pooled Degrees of Freedom: } 49 \\
\text { Standard Uncertainty: } 2.66 \cdot 10^{-6} \text { atom/atom }\end{array}$} & \\
\hline$\delta_{65 B C F D a y 1}:$ & \multicolumn{2}{|c|}{$\begin{array}{l}\text { Type B normal distribution } \\
\text { Value: } 1 \text { atom/atom } \\
\text { Expanded Uncertainty: } 0.00071524 \text { atom/atom } \\
\text { Coverage Factor: } 1\end{array}$} & \\
\hline \multirow[t]{9}{*}{$\mathbf{X}_{65 \text { Day2 }}$ : } & \multicolumn{2}{|c|}{$\begin{array}{l}\text { Type A } \\
\text { Method of observation: Direct } \\
\text { Number of observations: } 6\end{array}$} & \\
\hline & No. & Observation & \\
\hline & 1 & 0.015109 atom/atom & \\
\hline & 2 & 0.015142 atom/atom & \\
\hline & 3 & 0.015133 atom/atom & \\
\hline & 4 & 0.015152 atom/atom & \\
\hline & 5 & 0.015120 atom/atom & \\
\hline & 6 & 0.015129 atom/atom & \\
\hline & \multicolumn{2}{|c|}{$\begin{array}{l}\text { Type B normal distribution } \\
\text { Value: } 1 \text { atom/atom } \\
\text { Expanded Uncertainty: } 0.00024954 \text { atom/atom } \\
\text { Coverage Factor: } 1\end{array}$} & \\
\hline Date: $12 / 01 / 2010$ & \multicolumn{2}{|c|}{ File: PTRM10-1.1 11-29-10KLN.smu } & Page 4 of 8 \\
\hline
\end{tabular}


PNNL-20047

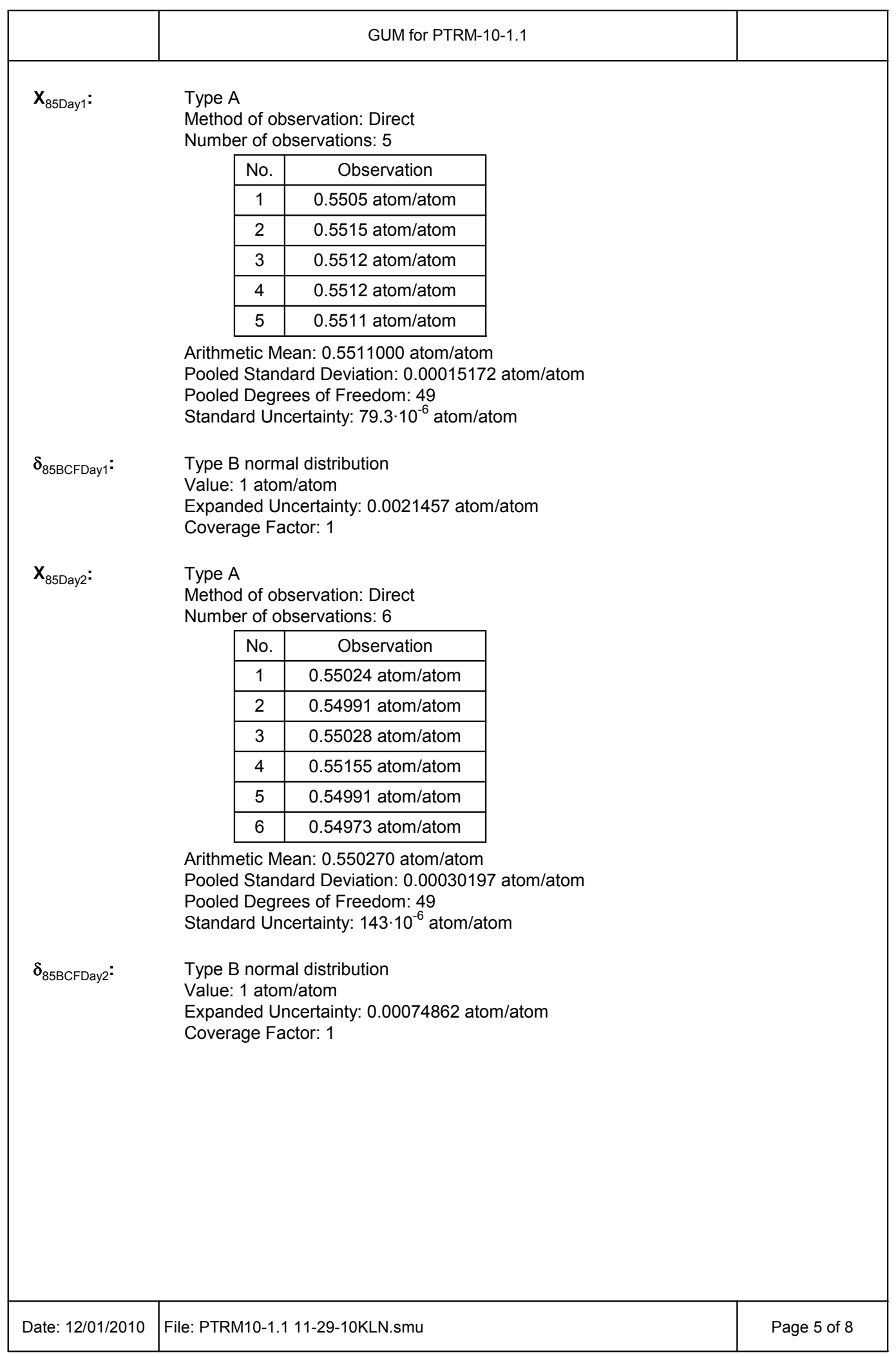

Generated with GUM Workbench Pro Version 2.4.1.332 


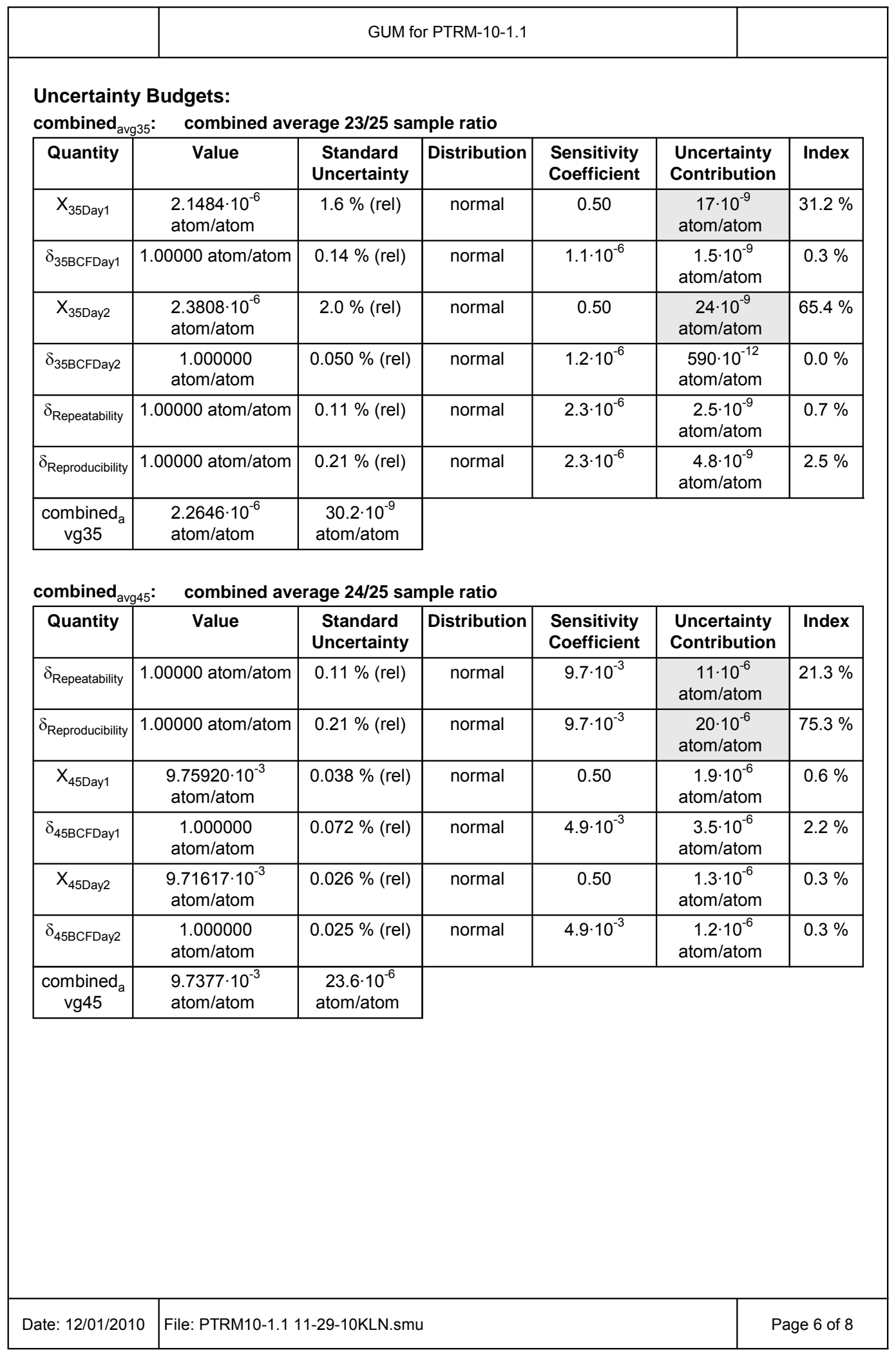




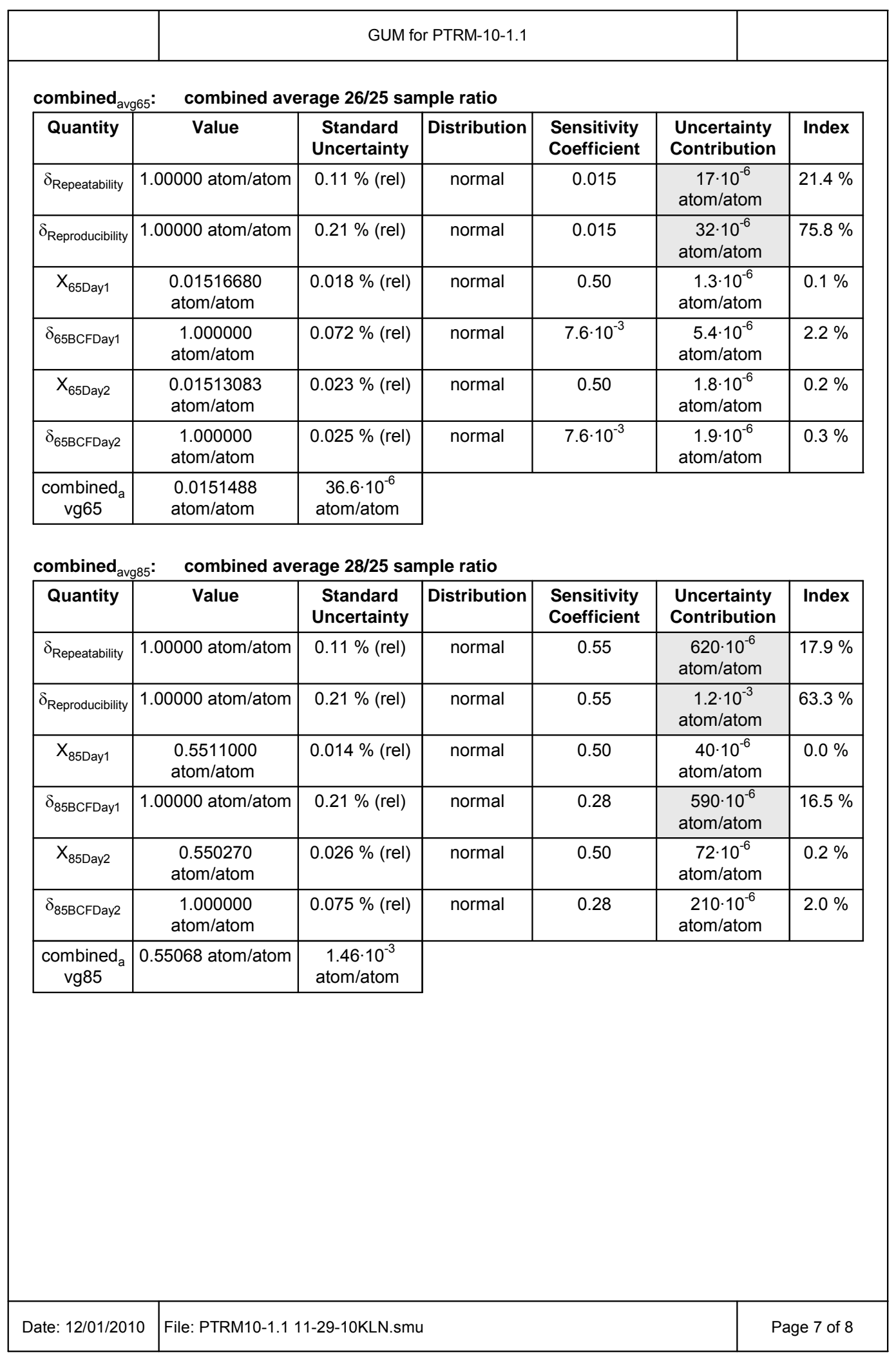


PNNL-20047

\begin{tabular}{|c|c|c|c|c|}
\hline & \multicolumn{3}{|c|}{ GUM for PTRM-10-1.1 } & \\
\hline Quantity & Value & $\begin{array}{l}\text { Expanded } \\
\text { Uncertainty }\end{array}$ & $\begin{array}{l}\text { Coverage } \\
\text { factor }\end{array}$ & Coverage \\
\hline combined $_{\text {avg } 35}$ & $2.265 \cdot 10^{-6}$ atom/atom & $\begin{array}{c}60 \cdot 10^{-9} \\
\text { atom/atom }\end{array}$ & 2.00 & $95 \%$ (normal) \\
\hline combined $_{\text {avg } 45}$ & $9.738 \cdot 10^{-3}$ atom/atom & $\begin{array}{c}47 \cdot 10^{-6} \\
\text { atom/atom }\end{array}$ & 2.00 & $95 \%$ (normal) \\
\hline combined $_{\text {avg65 }}$ & 0.015149 atom/atom & $\begin{array}{c}73 \cdot 10^{-6} \\
\text { atom/atom }\end{array}$ & 2.00 & $95 \%$ (normal) \\
\hline combined $_{\text {avg85 }}$ & 0.5507 atom/atom & $\begin{array}{c}2.9 \cdot 10^{-3} \\
\text { atom/atom }\end{array}$ & 2.00 & 95\% (normal) \\
\hline
\end{tabular}


Exhibit B.2: Uncertainty Budgets from PTRM10-1.2.

\begin{tabular}{|c|c|c|c|}
\hline & \multicolumn{3}{|c|}{ GUM for PTRM-10-1.2 } \\
\hline \multicolumn{4}{|c|}{ GUM for PTRM-10-1.2 } \\
\hline \multicolumn{4}{|l|}{ Author: d3m597 } \\
\hline \multicolumn{4}{|c|}{$\begin{array}{l}\text { Modified to simplify. RSD (or delta) values come from spreadsheet containing all CRM-129A data and } \\
\text { calculated individual BCF delta factors. }\end{array}$} \\
\hline $\begin{array}{r}\text { Model Equati } \\
\text { combinec } \\
\text { combinec } \\
\text { combine }\end{array}$ & $\begin{array}{l}\text { avg48 }=\left(\left(X_{48}\right.\right. \\
\operatorname{avg58}=\left(\left(X_{58}\right.\right. \\
\operatorname{avg68}=\left(\left(X_{68}\right.\right.\end{array}$ & $\begin{array}{l}\text { Day1 }{ }^{*} \delta_{48 \mathrm{BCFDay} 1}+\mathrm{X}_{48 \mathrm{D}} \\
\text { Day1 }{ }^{*} \delta_{58 \mathrm{BCFDay} 1}+\mathrm{X}_{58 \mathrm{D}} \\
{ }_{\text {Day1 }}{ }^{*} \delta_{68 \mathrm{BCFDay} 1}+\mathrm{X}_{68 \mathrm{D}}\end{array}$ & $\begin{array}{l}\text { bility; } \\
\text { bility; } \\
\text { bility; }\end{array}$ \\
\hline \multicolumn{4}{|c|}{ List of Quantities: } \\
\hline Quantity & Unit & Definition & \\
\hline combined $_{\text {avg } 48}$ & atom/atom & combined average $o$ & \\
\hline $\mathrm{X}_{48 \mathrm{Day} 1}$ & atom/atom & average value of $24 /$ & \\
\hline$\delta_{48 \mathrm{BCFDay} 1}$ & atom/atom & $\begin{array}{l}\text { delta factor for relati } \\
\text { for Day } 1\end{array}$ & $24 / 28$ ratio \\
\hline $\mathrm{X}_{48 \mathrm{Day} 2}$ & atom/atom & average value of $24 /$ & \\
\hline$\delta_{48 B C F D a y 2}$ & atom/atom & $\begin{array}{l}\text { delta factor for relati } \\
\text { for Day } 2\end{array}$ & $24 / 28$ ratio \\
\hline$\delta_{\text {Repeatability }}$ & atom/atom & $\begin{array}{l}\text { delta factor for relati } \\
25 / 28 \text { for control star }\end{array}$ & eatability of \\
\hline$\delta_{\text {Reproducibility }}$ & atom/atom & $\begin{array}{l}\text { delta factor for relati } \\
\text { of } 25 / 28 \text { for control } s\end{array}$ & producibility \\
\hline combined $_{\text {avg } 58}$ & atom/atom & combined average $o$ & \\
\hline$X_{58 \text { Day } 1}$ & atom/atom & average value of the & \\
\hline$\delta_{58 \mathrm{BCFDay} 1}$ & atom/atom & $\begin{array}{l}\text { delta factor for relati } \\
\text { for Day } 1\end{array}$ & $25 / 28$ ratio \\
\hline $\mathrm{X}_{58 \mathrm{Day} 2}$ & atom/atom & average value of the & \\
\hline$\delta_{58 \mathrm{BCFDay} 2}$ & atom/atom & $\begin{array}{l}\text { delta factor for relati } \\
\text { for Day } 2\end{array}$ & $25 / 28$ ratio \\
\hline combined $_{\text {avg } 68}$ & atom/atom & combined average o & \\
\hline $\mathrm{X}_{68 \mathrm{Day} 1}$ & atom/atom & average value of the & \\
\hline$\delta_{68 \text { BCFDay } 1}$ & atom/atom & $\begin{array}{l}\text { delta factor for relati } \\
\text { for Day } 1\end{array}$ & $26 / 28$ ratio \\
\hline$X_{68 \text { Day } 2}$ & atom/atom & average value of the & \\
\hline$\delta_{68 \text { BCFDay2 }}$ & atom/atom & $\begin{array}{l}\text { delta factor for relati } \\
\text { for Day } 2\end{array}$ & $26 / 28$ ratio \\
\hline Date: $12 / 01 / 2010$ & File: PTRM1 & 0-1.2 11-29-10KLN.smu & Page 1 of 6 \\
\hline
\end{tabular}




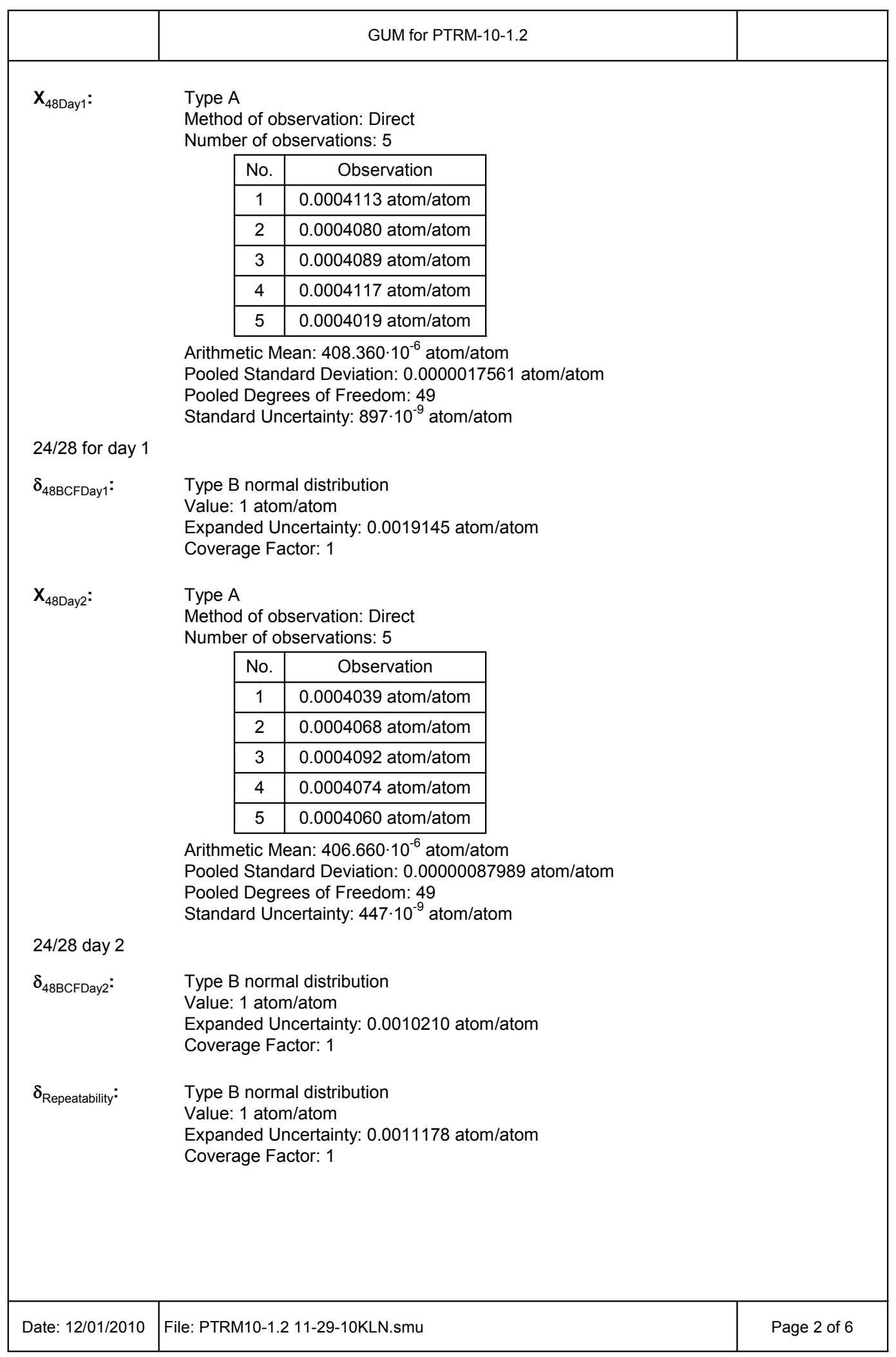


PNNL-20047

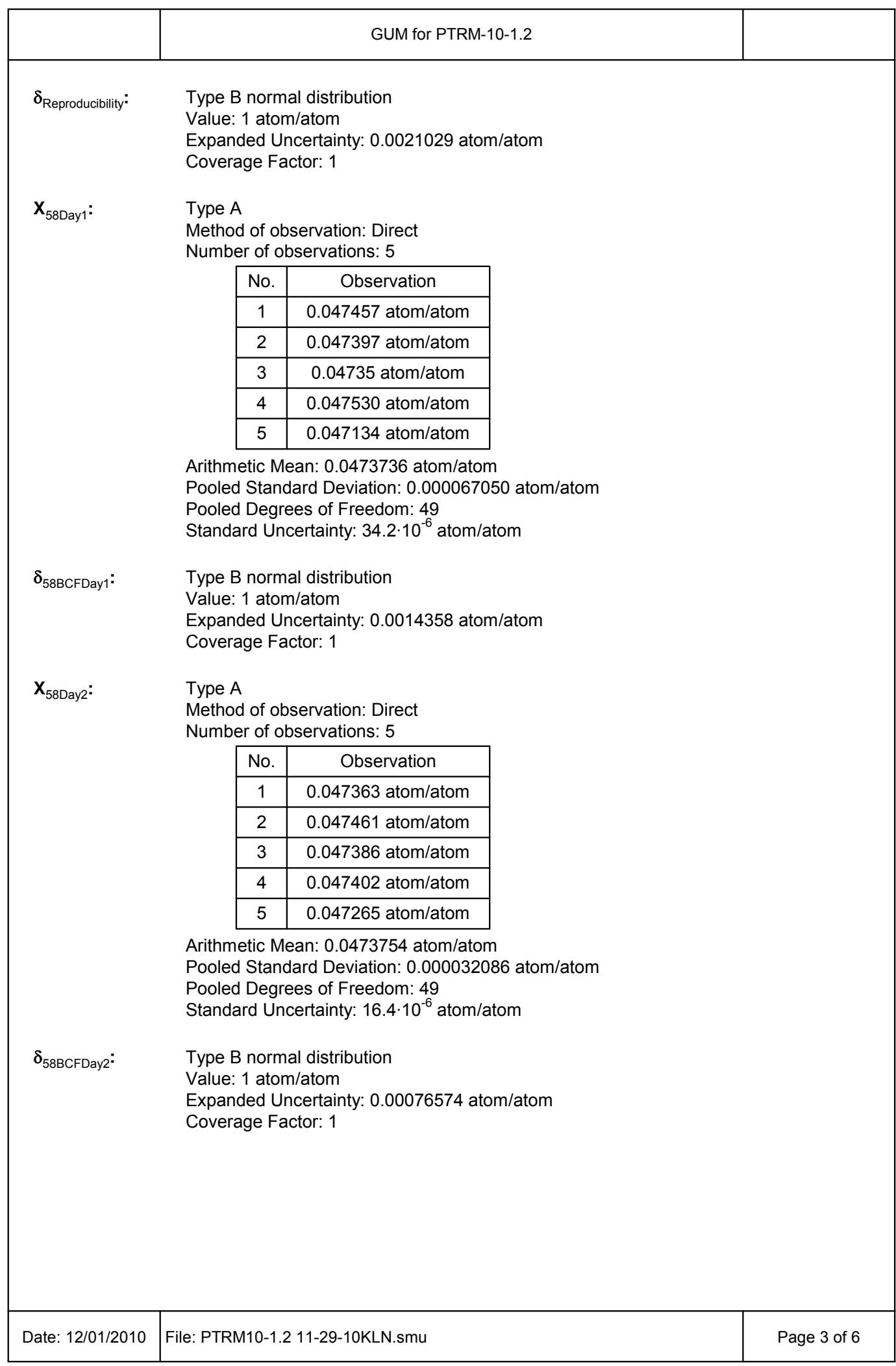


PNNL-20047

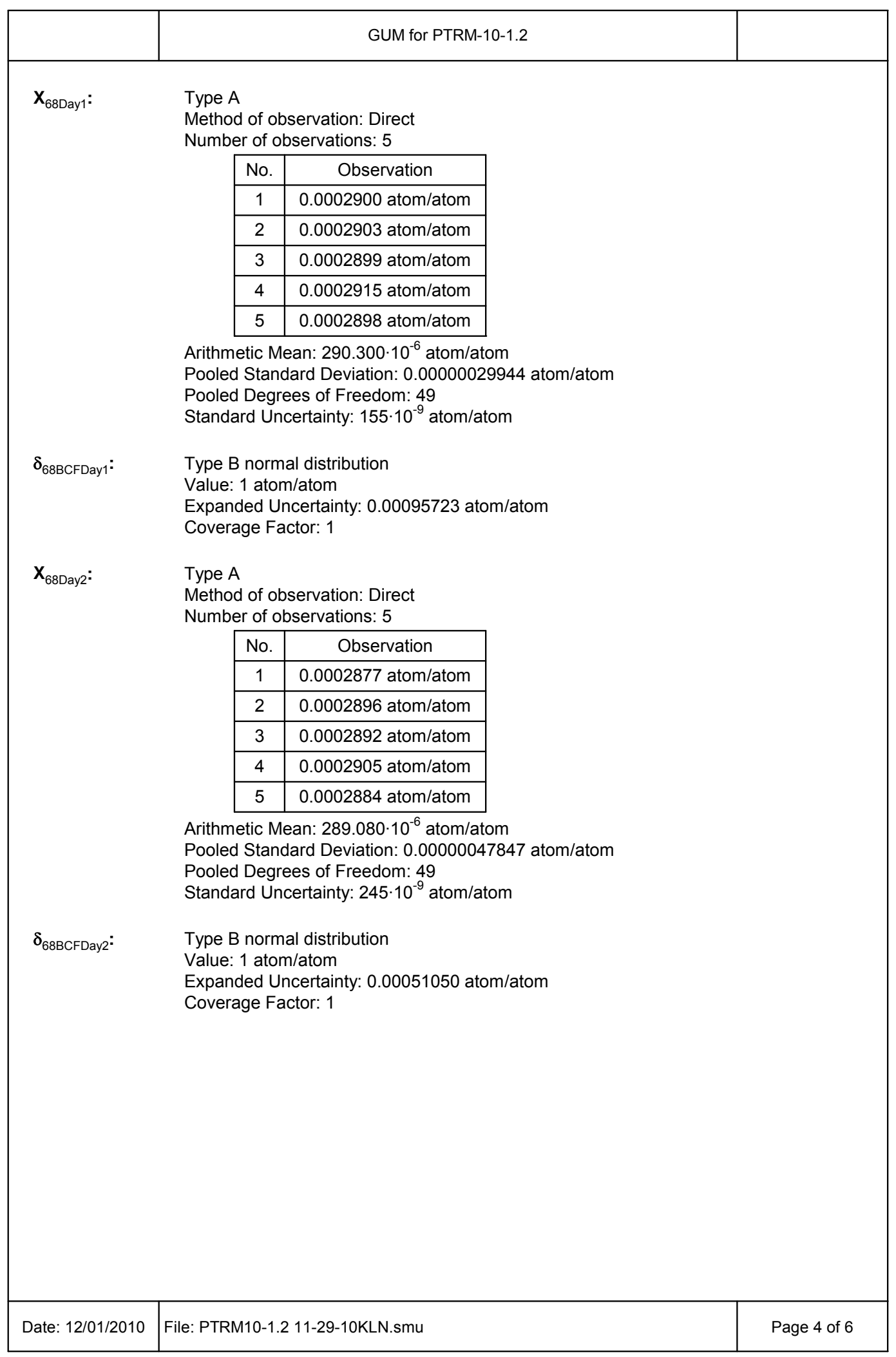




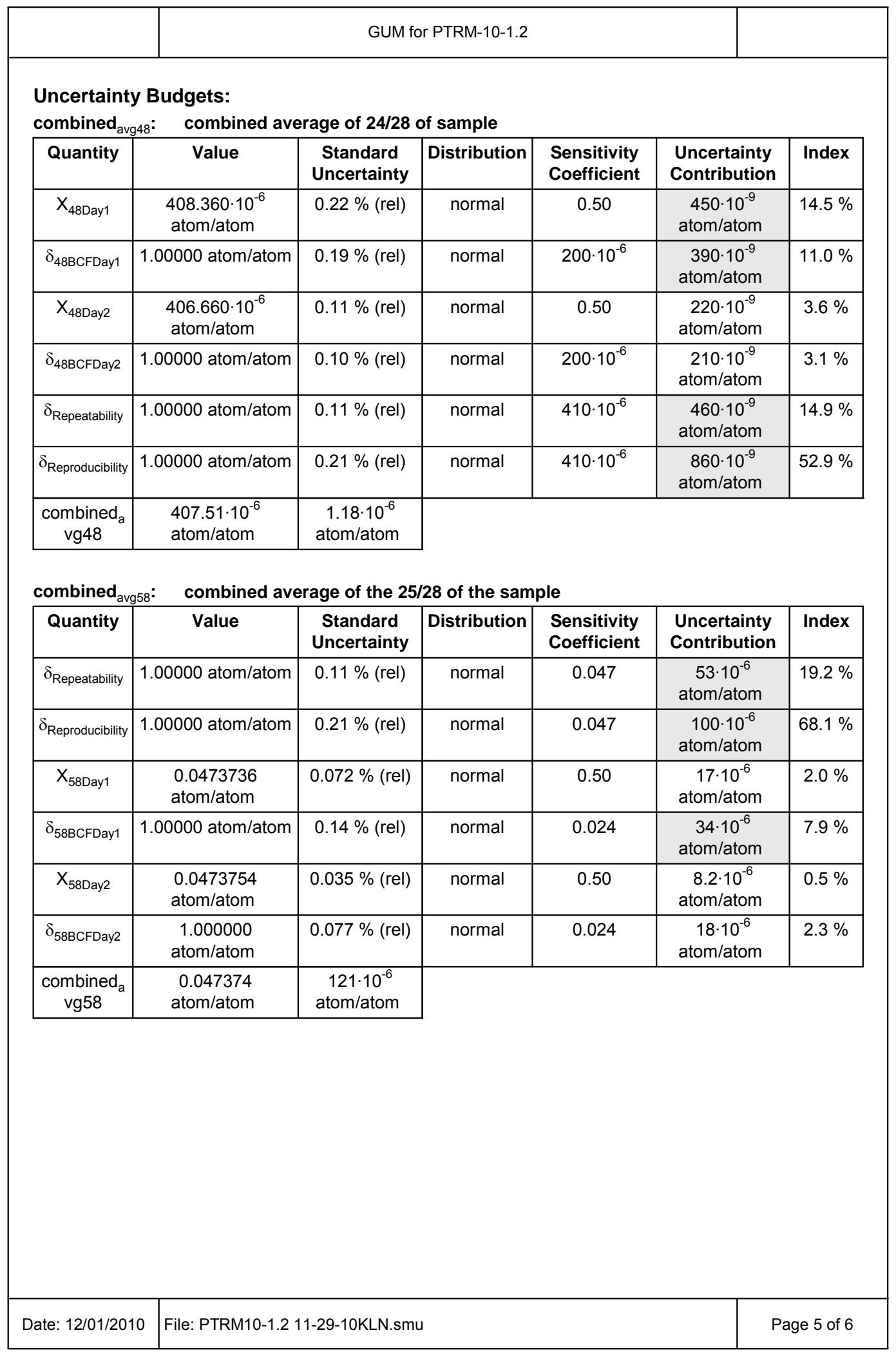




\begin{tabular}{|c|c|c|c|c|c|c|c|c|}
\hline & \multicolumn{6}{|c|}{ GUM for PTRM-10-1.2 } & & \\
\hline combined $_{\text {avg68: }}$ & \multicolumn{8}{|c|}{ combined average of the $26 / 28$ of the sample } \\
\hline Quantity & Value & \multicolumn{2}{|c|}{$\begin{array}{l}\text { Standard } \\
\text { Uncertainty }\end{array}$} & \multicolumn{2}{|c|}{ Distribution } & $\begin{array}{l}\text { Sensitivity } \\
\text { Coefficient }\end{array}$ & $\begin{array}{l}\text { Uncertainty } \\
\text { Contribution }\end{array}$ & Index \\
\hline$\delta_{\text {Repeatability }}$ & 1.00000 atom/atom & \multicolumn{2}{|c|}{$0.11 \%$ (rel) } & \multicolumn{2}{|c|}{ normal } & $290 \cdot 10^{-6}$ & $\begin{array}{c}320 \cdot 10^{-9} \\
\text { atom/atom }\end{array}$ & $20.1 \%$ \\
\hline \begin{tabular}{|l|l}
$\delta_{\text {Reproducibility }}$ & 1. \\
\end{tabular} & 1.00000 atom/atom & \multicolumn{2}{|c|}{$0.21 \%$ (rel) } & \multicolumn{2}{|c|}{ normal } & $290 \cdot 10^{-6}$ & $\begin{array}{c}610 \cdot 10^{-9} \\
\text { atom/atom }\end{array}$ & $71.1 \%$ \\
\hline$X_{68 D a y 1}$ & $\begin{array}{l}290.300 \cdot 10^{-6} \\
\text { atom/atom }\end{array}$ & \multicolumn{2}{|c|}{$0.053 \%$ (rel) } & \multicolumn{2}{|c|}{ normal } & 0.50 & $\begin{array}{c}77 \cdot 10^{-9} \\
\text { atom/atom }\end{array}$ & $1.1 \%$ \\
\hline$\delta_{68 B C F D a y 1}$ & $\begin{array}{r}1.000000 \\
\text { atom/atom }\end{array}$ & \multicolumn{2}{|c|}{$0.096 \%$ (rel) } & \multicolumn{2}{|c|}{ normal } & $150 \cdot 10^{-6}$ & $\begin{array}{c}140 \cdot 10^{-9} \\
\text { atom/atom }\end{array}$ & $3.7 \%$ \\
\hline $\mathrm{X}_{68 \mathrm{Day} 2}$ & $\begin{array}{l}289.080 \cdot 10^{-6} \\
\text { atom/atom }\end{array}$ & \multicolumn{2}{|c|}{$0.085 \%$ (rel) } & \multicolumn{2}{|c|}{ normal } & 0.50 & $\begin{array}{c}120 \cdot 10^{-9} \\
\text { atom/atom }\end{array}$ & $2.9 \%$ \\
\hline$\delta_{68 B C F D a y 2}$ & $\begin{array}{r}1.000000 \\
\text { atom/atom }\end{array}$ & \multicolumn{2}{|c|}{$0.051 \%$ (rel) } & \multicolumn{2}{|c|}{ normal } & $140 \cdot 10^{-6}$ & $\begin{array}{c}74 \cdot 10^{-9} \\
\text { atom/atom }\end{array}$ & $1.0 \%$ \\
\hline $\begin{array}{c}\text { combined }_{a} \\
\text { vg68 }\end{array}$ & $\begin{array}{l}289.690 \cdot 10^{-6} \\
\text { atom/atom }\end{array}$ & \multicolumn{2}{|c|}{$\begin{array}{l}722 \cdot 10^{-9} \\
\text { atom/atom }\end{array}$} & & & & & \\
\hline \multicolumn{9}{|l|}{ Results: } \\
\hline Quantity & \multicolumn{2}{|l|}{ Value } & \multicolumn{2}{|c|}{$\begin{array}{l}\text { Expanded } \\
\text { Uncertainty }\end{array}$} & & $\begin{array}{l}\text { overage } \\
\text { factor }\end{array}$ & \multicolumn{2}{|l|}{ Coverage } \\
\hline combined $_{\text {avg } 48}$ & \multicolumn{2}{|c|}{$407.5 \cdot 10^{-6}$ atom/atom } & \multicolumn{2}{|c|}{$\begin{array}{c}2.4 \cdot 10^{-6} \\
\text { atom/atom }\end{array}$} & & 2.00 & \multicolumn{2}{|c|}{ 95\% (t-table $95.45 \%$ ) } \\
\hline combined $_{\text {avg58 }}$ & \multicolumn{2}{|c|}{0.04737 atom/atom } & \multicolumn{2}{|c|}{$\begin{array}{c}240 \cdot 10^{-6} \\
\text { atom/atom }\end{array}$} & & 2.00 & \multicolumn{2}{|c|}{ 95\% (t-table $95.45 \%$ ) } \\
\hline combined $_{\text {avg } 68}$ & $289.7 \cdot 10^{-6}$ atom/ & atom & $\begin{array}{r}1.4 \\
\text { atom }\end{array}$ & $\begin{array}{l}10^{-6} \\
\text { atom }\end{array}$ & & 2.00 & 95\% (t-table 95.4 & $5 \%)$ \\
\hline
\end{tabular}


Exhibit B.3: Uncertainty Budgets from PTRM10-3.1.

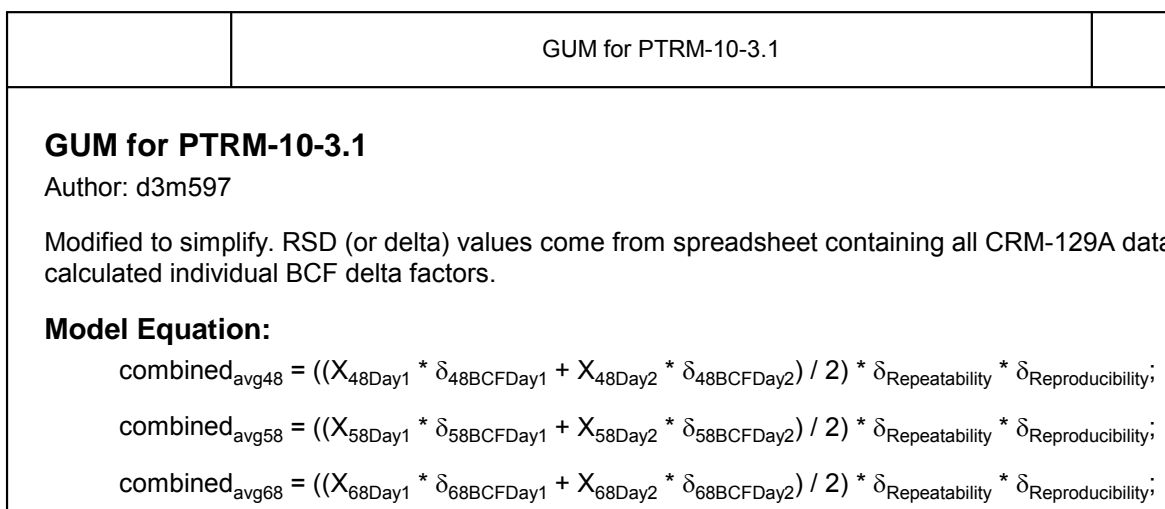

List of Quantities:

\begin{tabular}{|c|c|c|}
\hline Quantity & Unit & Definition \\
\hline combined $_{\text {avg } 48}$ & atom/atom & combined average of $24 / 28$ of sample \\
\hline $\mathrm{X}_{48 \mathrm{Day} 1}$ & atom/atom & average value of $24 / 28$ of sample for day 1 \\
\hline$\delta_{48 B C F D a y 1}$ & atom/atom & $\begin{array}{l}\text { delta factor for relative uncertainty for bias correction factor for } 24 / 28 \text { ratio } \\
\text { for Day } 1\end{array}$ \\
\hline $\mathrm{X}_{48 \mathrm{Day} 2}$ & atom/atom & average value of $24 / 28$ of sample for day 2 \\
\hline$\delta_{48 B C F D a y 2}$ & atom/atom & $\begin{array}{l}\text { delta factor for relative uncertainty for bias correction factor for } 24 / 28 \text { ratio } \\
\text { for Day } 1\end{array}$ \\
\hline$\delta_{\text {Repeatability }}$ & atom/atom & $\begin{array}{l}\text { delta factor for relative uncertainty characterizing intra-day repeatability of } \\
25 / 28 \text { for control standard }\end{array}$ \\
\hline$\delta_{\text {Reproducibility }}$ & atom/atom & $\begin{array}{l}\text { delta factor for relative uncertainty characterizing day-to-day reproducibility } \\
\text { of } 25 / 28 \text { for control standard }\end{array}$ \\
\hline combined $_{\text {avg } 58}$ & atom/atom & combined average of the $25 / 28$ of the sample \\
\hline $\mathrm{X}_{58 \mathrm{Day} 1}$ & atom/atom & average value of the $25 / 28$ of sample for day 1 \\
\hline$\delta_{58 \mathrm{BCFDay} 1}$ & atom/atom & $\begin{array}{l}\text { delta factor for relative uncertainty for bias correction factor for } 25 / 28 \text { ratio } \\
\text { for Day } 1\end{array}$ \\
\hline $\mathrm{X}_{58 \mathrm{Day} 2}$ & atom/atom & average value of the $25 / 28$ of sample for day 2 \\
\hline$\delta_{58 \mathrm{BCFDay} 2}$ & atom/atom & $\begin{array}{l}\text { delta factor for relative uncertainty for bias correction factor for } 25 / 28 \text { ratio } \\
\text { for Day } 2\end{array}$ \\
\hline combined $_{\text {avg68 }}$ & atom/atom & combined average of the $26 / 28$ of the sample \\
\hline $\mathrm{X}_{68 \text { Day1 }}$ & atom/atom & average value of the $26 / 28$ of sample for day 1 \\
\hline$\delta_{68 B C F D a y 1}$ & atom/atom & $\begin{array}{l}\text { delta factor for relative uncertainty for bias correction factor for } 26 / 28 \text { ratio } \\
\text { for Day } 1\end{array}$ \\
\hline $\mathrm{X}_{68 \mathrm{Day} 2}$ & atom/atom & average value of the $26 / 28$ of sample for day 2 \\
\hline$\delta_{68 \text { BCFDay } 2}$ & atom/atom & $\begin{array}{l}\text { delta factor for relative uncertainty for bias correction factor for } 26 / 28 \text { ratio } \\
\text { for Day } 2\end{array}$ \\
\hline
\end{tabular}




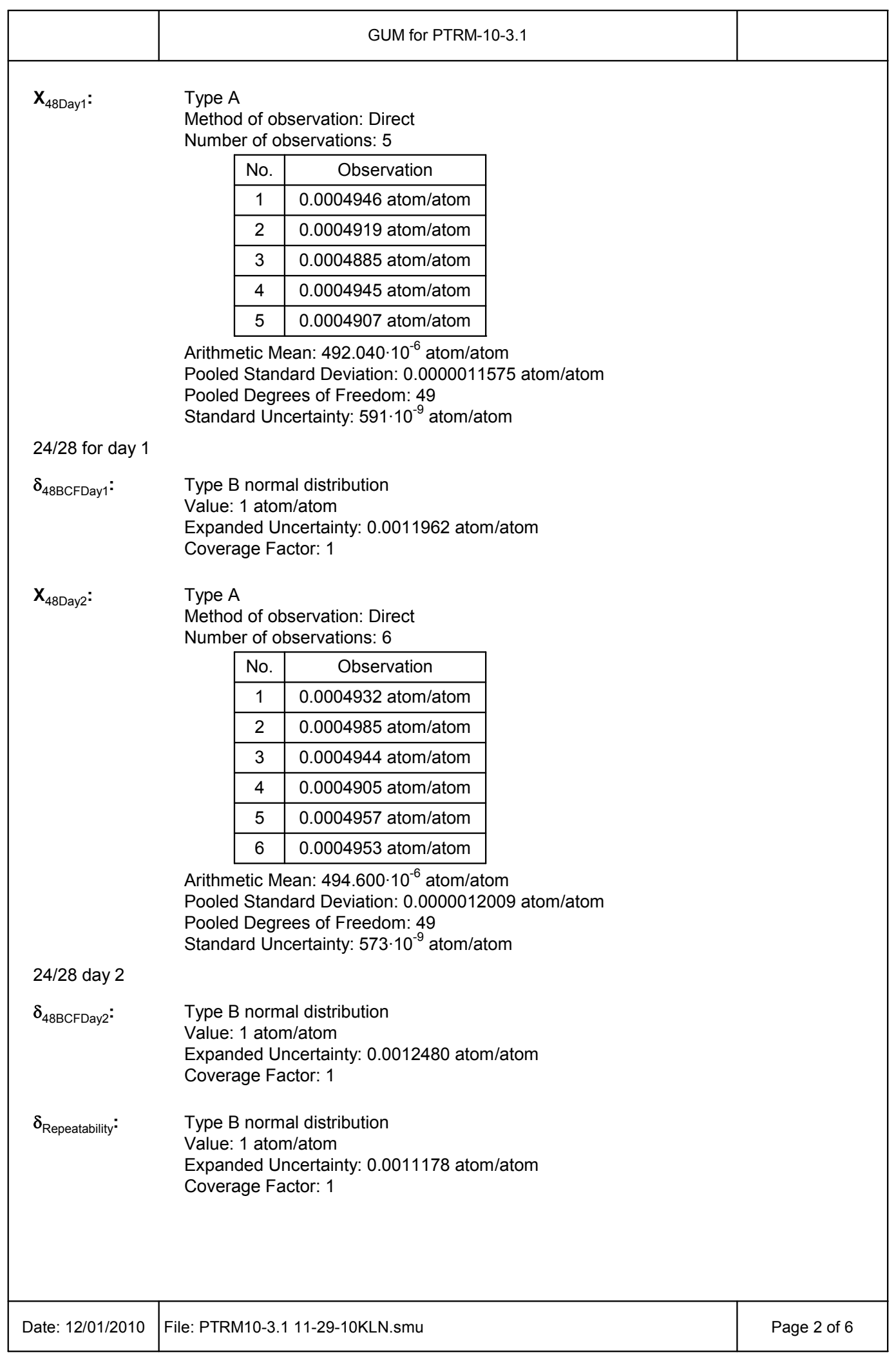


PNNL-20047

\begin{tabular}{|c|c|c|c|}
\hline \multicolumn{3}{|r|}{ GUM for PTRM-10-3.1 } & \\
\hline$\delta_{\text {Reproducibility }}:$ & \multicolumn{2}{|c|}{$\begin{array}{l}\text { Type B normal distribution } \\
\text { Value: } 1 \text { atom/atom } \\
\text { Expanded Uncertainty: } 0.0021029 \text { atom/atom } \\
\text { Coverage Factor: } 1\end{array}$} & \\
\hline \multirow[t]{8}{*}{$\mathbf{X}_{58 \text { Day1 }}$ : } & \multicolumn{2}{|c|}{$\begin{array}{l}\text { Type A } \\
\text { Method of observation: Direct } \\
\text { Number of observations: } 5\end{array}$} & \\
\hline & No. & Observation & \\
\hline & 1 & 0.052825 atom/atom & \\
\hline & 2 & 0.05260 atom/atom & \\
\hline & 3 & 0.052737 atom/atom & \\
\hline & 4 & 0.052800 atom/atom & \\
\hline & 5 & 0.052760 atom/atom & \\
\hline & \multicolumn{2}{|c|}{$\begin{array}{l}\text { Arithmetic Mean: } 0.0527444 \text { atom/atom } \\
\text { Pooled Standard Deviation: } 0.000038588 \text { atom/atom } \\
\text { Pooled Degrees of Freedom: } 49 \\
\text { Standard Uncertainty: } 19.8 \cdot 10^{-6} \text { atom/atom }\end{array}$} & \\
\hline$\delta_{\text {58BCFDay } 1}:$ & \multicolumn{2}{|c|}{$\begin{array}{l}\text { Type B normal distribution } \\
\text { Value: } 1 \text { atom/atom } \\
\text { Expanded Uncertainty: } 0.00089717 \text { atom/atom } \\
\text { Coverage Factor: } 1\end{array}$} & \\
\hline \multirow[t]{8}{*}{$\mathbf{X}_{58 \text { Day2 }}:$} & \multicolumn{2}{|c|}{$\begin{array}{l}\text { Type A } \\
\text { Method of observation: Direct } \\
\text { Number of observations: } 6\end{array}$} & \\
\hline & No. & Observation & \\
\hline & 1 & 0.05301 atom/atom & \\
\hline & 2 & 0.052921 atom/atom & \\
\hline & 3 & 0.052978 atom/atom & \\
\hline & 4 & 0.052874 atom/atom & \\
\hline & 5 & 0.053020 atom/atom & \\
\hline & 6 & 0.05302 atom/atom & \\
\hline \multirow[b]{2}{*}{$\delta_{58 B C F D a y 2}:$} & \multicolumn{2}{|c|}{$\begin{array}{l}\text { Arithmetic Mean: } 0.0529705 \text { atom/atom } \\
\text { Pooled Standard Deviation: } 0.000027173 \text { atom/atom } \\
\text { Pooled Degrees of Freedom: } 49 \\
\text { Standard Uncertainty: } 13.0 \cdot 10^{-6} \text { atom/atom }\end{array}$} & \\
\hline & \multicolumn{2}{|c|}{$\begin{array}{l}\text { Type B normal distribution } \\
\text { Value: } 1 \text { atom/atom } \\
\text { Expanded Uncertainty: } 0.00093599 \text { atom/atom } \\
\text { Coverage Factor: } 1\end{array}$} & \\
\hline Date: $12 / 01 / 2010$ & \multicolumn{2}{|c|}{ File: PTRM10-3.1 11-29-10KLN.smu } & Page 3 of 6 \\
\hline
\end{tabular}


PNNL-20047

\begin{tabular}{|c|c|c|c|}
\hline & \multicolumn{2}{|r|}{ GUM for PTRM-10-3.1 } & \\
\hline \multirow[t]{8}{*}{$\mathrm{X}_{68 \text { Day } 1}:$} & \multicolumn{2}{|c|}{$\begin{array}{l}\text { Type A } \\
\text { Method of observation: Direct } \\
\text { Number of observations: } 5\end{array}$} & \\
\hline & No. & Observation & \\
\hline & 1 & 0.0001583 atom/atom & \\
\hline & 2 & 0.0001590 atom/atom & \\
\hline & 3 & 0.0001597 atom/atom & \\
\hline & 4 & 0.0001580 atom/atom & \\
\hline & 5 & 0.0001590 atom/atom & \\
\hline & \multicolumn{2}{|c|}{$\begin{array}{l}\text { Arithmetic Mean: } 158.800 \cdot 10^{-6} \text { atom/atom } \\
\text { Pooled Standard Deviation: } 0.00000030074 \text { atom/atom } \\
\text { Pooled Degrees of Freedom: } 49 \\
\text { Standard Uncertainty: } 153 \cdot 10^{-9} \text { atom/atom }\end{array}$} & \\
\hline$\delta_{68 B C F D a y 1}:$ & \multicolumn{2}{|c|}{$\begin{array}{l}\text { Type B normal distribution } \\
\text { Value: } 1 \text { atom/atom } \\
\text { Expanded Uncertainty: } 0.00059812 \text { atom/atom } \\
\text { Coverage Factor: } 1\end{array}$} & \\
\hline \multirow[t]{9}{*}{$\mathbf{X}_{68 \text { Day2 }}$} & \multicolumn{2}{|c|}{$\begin{array}{l}\text { Type A } \\
\text { Method of observation: Direct } \\
\text { Number of observations: } 6\end{array}$} & \\
\hline & No. & Observation & \\
\hline & 1 & 0.0001599 atom/atom & \\
\hline & 2 & 0.0001582 atom/atom & \\
\hline & 3 & 0.0001595 atom/atom & \\
\hline & 4 & 0.0001586 atom/atom & \\
\hline & 5 & 0.0001611 atom/atom & \\
\hline & 6 & 0.0001602 atom/atom & \\
\hline & \multicolumn{2}{|c|}{$\begin{array}{l}\text { Arithmetic Mean: } 159.583 \cdot 10^{-6} \text { atom/atom } \\
\text { Pooled Standard Deviation: } 0.00000047396 \text { atom/atom } \\
\text { Pooled Degrees of Freedom: } 49 \\
\text { Standard Uncertainty: } 227 \cdot 10^{-9} \text { atom/atom }\end{array}$} & \\
\hline$\delta_{68 \mathrm{BCFDay2}}:$ & \multicolumn{2}{|c|}{$\begin{array}{l}\text { Type B normal distribution } \\
\text { Value: } 1 \text { atom/atom } \\
\text { Expanded Uncertainty: } 0.00062399 \text { atom/atom } \\
\text { Coverage Factor: } 1\end{array}$} & \\
\hline Date: $12 / 01 / 2010$ & \multicolumn{2}{|c|}{ File: PTRM10-3.1 11-29-10KLN.smu } & Page 4 of 6 \\
\hline
\end{tabular}




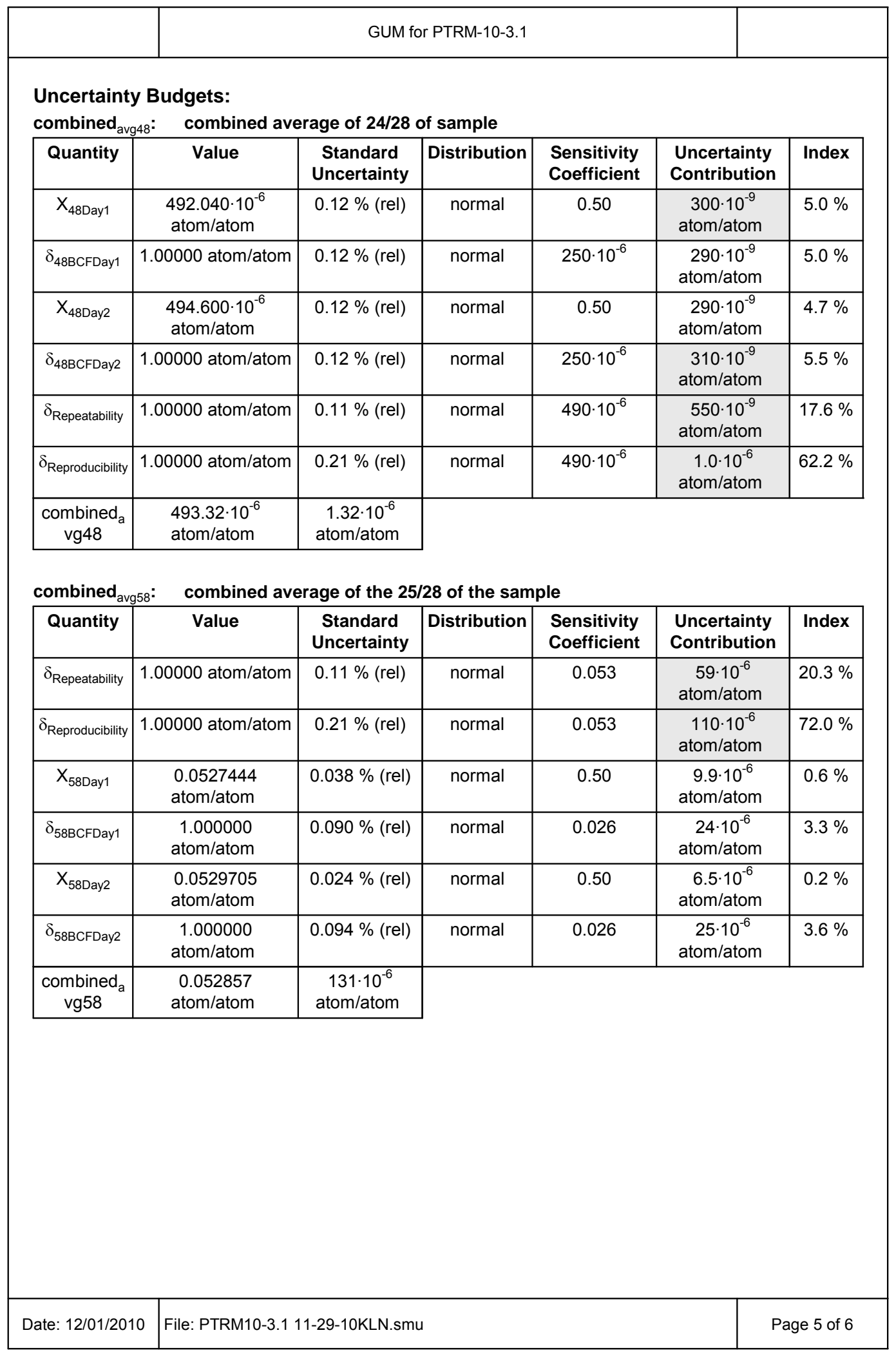




\begin{tabular}{|c|c|c|c|c|c|c|c|c|}
\hline & \multicolumn{6}{|c|}{ GUM for PTRM-10-3.1 } & & \\
\hline combined $_{\text {avg68: }}$ & \multicolumn{8}{|c|}{ combined average of the $26 / 28$ of the sample } \\
\hline Quantity & Value & \multicolumn{2}{|c|}{$\begin{array}{l}\text { Standard } \\
\text { Uncertainty }\end{array}$} & \multicolumn{2}{|c|}{ Distribution } & $\begin{array}{l}\text { Sensitivity } \\
\text { Coefficient }\end{array}$ & $\begin{array}{l}\text { Uncertainty } \\
\text { Contribution }\end{array}$ & Index \\
\hline$\delta_{\text {Repeatability }}$ & 1.00000 atom/atom & \multicolumn{2}{|c|}{$0.11 \%$ (rel) } & \multicolumn{2}{|c|}{ normal } & $160 \cdot 10^{-6}$ & $\begin{array}{c}180 \cdot 10^{-9} \\
\text { atom/atom }\end{array}$ & $18.9 \%$ \\
\hline \begin{tabular}{|l|l|}
$\delta_{\text {Reproducibility }}$ & 1. \\
\end{tabular} & 1.00000 atom/atom & \multicolumn{2}{|c|}{$0.21 \%$ (rel) } & \multicolumn{2}{|c|}{ normal } & $160 \cdot 10^{-6}$ & $\begin{array}{c}330 \cdot 10^{-9} \\
\text { atom/atom }\end{array}$ & $67.0 \%$ \\
\hline$X_{68 D a y 1}$ & $\begin{array}{l}158.800 \cdot 10^{-6} \\
\text { atom/atom }\end{array}$ & \multicolumn{2}{|c|}{$0.096 \%$ (rel) } & \multicolumn{2}{|c|}{ normal } & 0.50 & $\begin{array}{c}77 \cdot 10^{-9} \\
\text { atom/atom }\end{array}$ & $3.5 \%$ \\
\hline$\delta_{68 B C F D a y 1}$ & $\begin{array}{r}1.000000 \\
\text { atom/atom }\end{array}$ & \multicolumn{2}{|c|}{$0.060 \%$ (rel) } & \multicolumn{2}{|c|}{ normal } & $79 \cdot 10^{-6}$ & $\begin{array}{c}47 \cdot 10^{-9} \\
\text { atom/atom }\end{array}$ & $1.3 \%$ \\
\hline $\mathrm{X}_{68 \mathrm{Day} 2}$ & $\begin{array}{l}159.583 \cdot 10^{-6} \\
\text { atom/atom }\end{array}$ & \multicolumn{2}{|c|}{$0.14 \%$ (rel) } & \multicolumn{2}{|c|}{ normal } & 0.50 & $\begin{array}{l}110 \cdot 10^{-9} \\
\text { atom/atom }\end{array}$ & $7.7 \%$ \\
\hline$\delta_{68 B C F D a y 2}$ & $\begin{array}{r}1.000000 \\
\text { atom/atom }\end{array}$ & \multicolumn{2}{|c|}{$0.062 \%$ (rel) } & \multicolumn{2}{|c|}{ normal } & $80 \cdot 10^{-6}$ & $\begin{array}{l}50 \cdot 10^{-9} \\
\text { atom/atom }\end{array}$ & $1.5 \%$ \\
\hline $\begin{array}{c}\text { combined }_{a} \\
\text { vg68 }\end{array}$ & $\begin{array}{l}159.192 \cdot 10^{-6} \\
\text { atom/atom }\end{array}$ & \multicolumn{2}{|c|}{$\begin{array}{c}409 \cdot 10^{-9} \\
\text { atom/atom }\end{array}$} & & & & & \\
\hline \multicolumn{9}{|l|}{ Results: } \\
\hline Quantity & \multicolumn{2}{|l|}{ Value } & \multicolumn{2}{|c|}{$\begin{array}{l}\text { Expanded } \\
\text { Uncertainty }\end{array}$} & & $\begin{array}{l}\text { overage } \\
\text { factor }\end{array}$ & \multicolumn{2}{|l|}{ Coverage } \\
\hline combined $_{\text {avg } 48}$ & \multicolumn{2}{|c|}{$493.3 \cdot 10^{-6}$ atom/atom } & \multicolumn{2}{|c|}{$\begin{array}{c}2.6 \cdot 10^{-6} \\
\text { atom/atom }\end{array}$} & & 2.00 & \multicolumn{2}{|c|}{ 95\% (t-table $95.45 \%$ ) } \\
\hline combined $_{\text {avg58 }}$ & \multicolumn{2}{|c|}{0.05286 atom/atom } & \multicolumn{2}{|c|}{$\begin{array}{c}260 \cdot 10^{-6} \\
\text { atom/atom }\end{array}$} & & 2.00 & \multicolumn{2}{|c|}{ 95\% (t-table $95.45 \%$ ) } \\
\hline combined $_{\text {avg } 68}$ & $159.19 \cdot 10^{-6}$ atom & latom & $\begin{array}{r}820 \\
\text { atom }\end{array}$ & $\begin{array}{l}\cdot 10^{-9} \\
\text { latom }\end{array}$ & & 2.00 & 95\% (t-table 95.4 & $5 \%)$ \\
\hline
\end{tabular}


Exhibit B.4: Uncertainty Budgets from PTRM10-3.2.

\begin{tabular}{|c|c|c|}
\hline & \multicolumn{2}{|c|}{ GUM for PTRM-10-3.2 } \\
\hline \multicolumn{3}{|c|}{ GUM for PTRM-10-3.2 } \\
\hline \multicolumn{3}{|c|}{ Author: d3m597 } \\
\hline \multicolumn{3}{|c|}{$\begin{array}{l}\text { Modified to simplify. RSD (or delta) values come from spreadsheet containing all CRM-129A data and } \\
\text { calculated individual BCF delta factors. }\end{array}$} \\
\hline \multirow{4}{*}{\multicolumn{3}{|c|}{$\begin{array}{l}\text { Model Equation: } \\
\begin{aligned} \text { combined }_{\text {avg } 48} & =\left(\left(\mathrm{X}_{48 \mathrm{Day} 1}{ }^{*} \delta_{48 \mathrm{BCFDay} 1}+\mathrm{X}_{48 \mathrm{Day} 2}{ }^{*} \delta_{48 \mathrm{BCFDay} 2}\right) / 2\right){ }^{*} \delta_{\text {Repeatability }}{ }^{*} \delta_{\text {Reproducibility; }} ; \\
\text { combined }_{\text {avg } 58} & =\left(\left(\mathrm{X}_{58 \mathrm{Day} 1}{ }^{*} \delta_{58 \mathrm{BCFDay} 1}+\mathrm{X}_{58 \mathrm{Day} 2}{ }^{*} \delta_{58 \mathrm{BCFDay} 2}\right) / 2\right){ }^{*} \delta_{\text {Repeatability }}{ }^{*} \delta_{\text {Reproducibility }} ; \\
\text { combined }_{\text {avg68 }} & =\left(\left(\mathrm{X}_{68 \mathrm{Day} 1}{ }^{*} \delta_{68 \mathrm{BCFDay} 1}+\mathrm{X}_{68 \mathrm{Day} 2}{ }^{*} \delta_{68 \mathrm{BCFDay} 2}\right) / 2\right){ }^{*} \delta_{\text {Repeatability }}{ }^{*} \delta_{\text {Reproducibility }} ;\end{aligned}\end{array}$}} \\
\hline & & \\
\hline & & \\
\hline & & \\
\hline \multicolumn{3}{|c|}{ List of Quantities: } \\
\hline Quantity & Unit & Definition \\
\hline combined $_{\text {avg } 48}$ & atom/atom & combined average of $24 / 28$ of sample \\
\hline $\mathrm{X}_{48 \mathrm{Day} 1}$ & atom/atom & average value of $24 / 28$ of sample for day 1 \\
\hline$\delta_{48 \mathrm{BCFDay} 1}$ & atom/atom & $\begin{array}{l}\text { delta factor for relative uncertainty for bias correction factor for } 24 / 28 \text { ratio } \\
\text { for Day } 1\end{array}$ \\
\hline $\mathrm{X}_{48 \mathrm{Day} 2}$ & atom/atom & average value of $24 / 28$ of sample for day 2 \\
\hline$\delta_{48 \mathrm{BCFDay} 2}$ & atom/atom & $\begin{array}{l}\text { delta factor for relative uncertainty for bias correction factor for } 24 / 28 \text { ratio } \\
\text { for Day } 2\end{array}$ \\
\hline$\delta_{\text {Repeatability }}$ & atom/atom & $\begin{array}{l}\text { delta factor for relative uncertainty characterizing intra-day repeatability of } \\
25 / 28 \text { for control standard }\end{array}$ \\
\hline$\delta_{\text {Reproducibility }}$ & atom/atom & $\begin{array}{l}\text { delta factor for relative uncertainty characterizing day-to-day reproducibility } \\
\text { of } 25 / 28 \text { for control standard }\end{array}$ \\
\hline combined $_{\text {avg } 58}$ & atom/atom & combined average of the $25 / 28$ of the sample \\
\hline $\mathrm{X}_{58 \mathrm{Day} 1}$ & atom/atom & average value of the $25 / 28$ of sample for day 1 \\
\hline$\delta_{58 \mathrm{BCFDay} 1}$ & atom/atom & $\begin{array}{l}\text { delta factor for relative uncertainty for bias correction factor for } 25 / 28 \text { ratio } \\
\text { for Day } 1\end{array}$ \\
\hline$X_{58 \text { Day2 }}$ & atom/atom & average value of the $25 / 28$ of sample for day 2 \\
\hline$\delta_{58 \mathrm{BCFDay} 2}$ & atom/atom & $\begin{array}{l}\text { delta factor for relative uncertainty for bias correction factor for } 25 / 28 \text { ratio } \\
\text { for Day } 2\end{array}$ \\
\hline combined $_{\text {avg68 }}$ & atom/atom & combined average of the $26 / 28$ of the sample \\
\hline $\mathrm{X}_{68 \mathrm{Day} 1}$ & atom/atom & average value of the $26 / 28$ of sample for day 1 \\
\hline$\delta_{68 \mathrm{BCFDay} 1}$ & atom/atom & $\begin{array}{l}\text { delta factor for relative uncertainty for bias correction factor for } 26 / 28 \text { ratio } \\
\text { for Day } 1\end{array}$ \\
\hline $\mathrm{X}_{68 \mathrm{Day} 2}$ & atom/atom & average value of the $26 / 28$ of sample for day 2 \\
\hline$\delta_{68 \mathrm{BCFDay} 2}$ & atom/atom & $\begin{array}{l}\text { delta factor for relative uncertainty for bias correction factor for } 26 / 28 \text { ratio } \\
\text { for Day } 2\end{array}$ \\
\hline Date: $12 / 01 / 2010$ & File: PTRM1C & 0-3.2 11-29-10KLN.smu \\
\hline
\end{tabular}




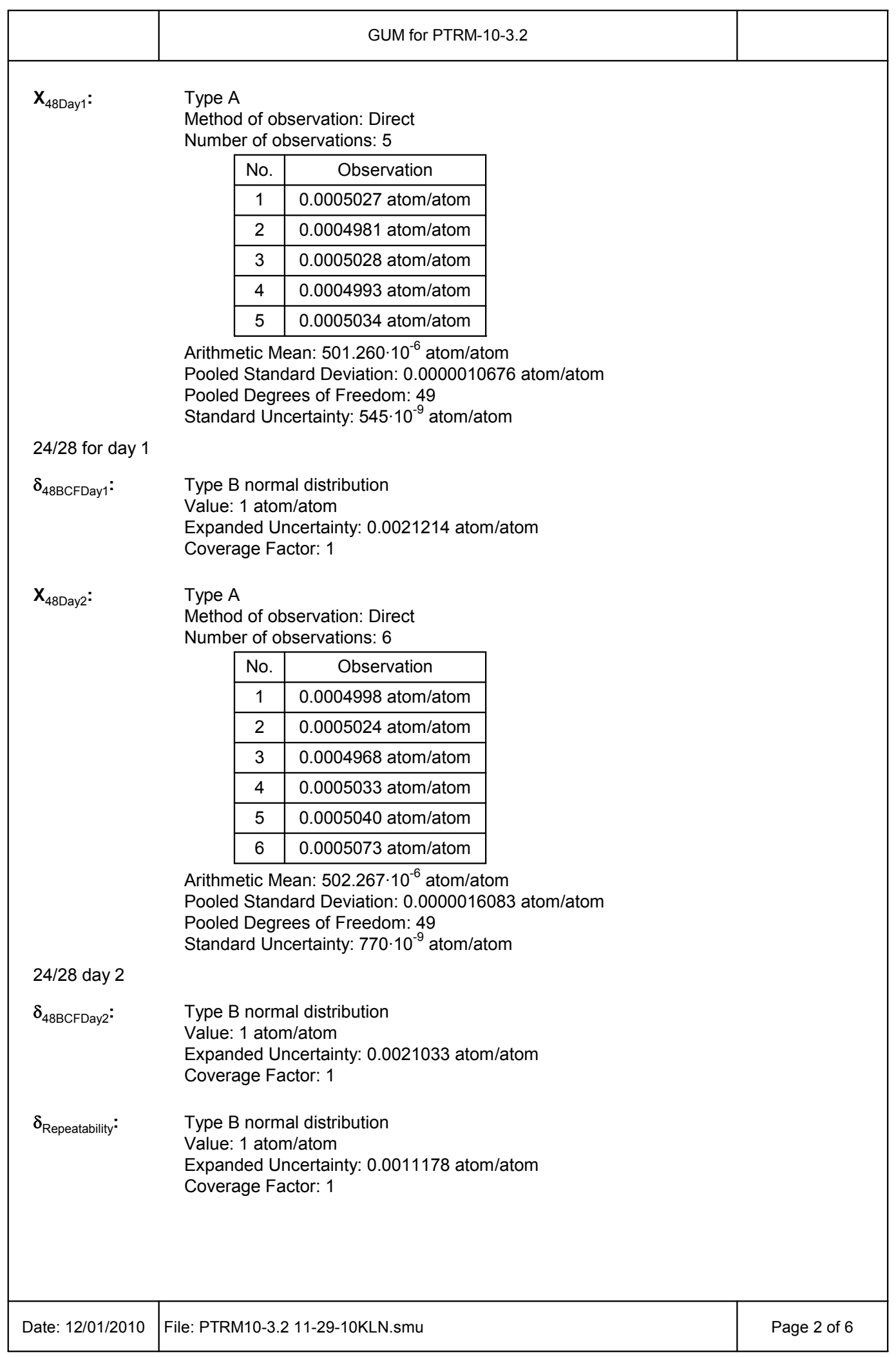


PNNL-20047

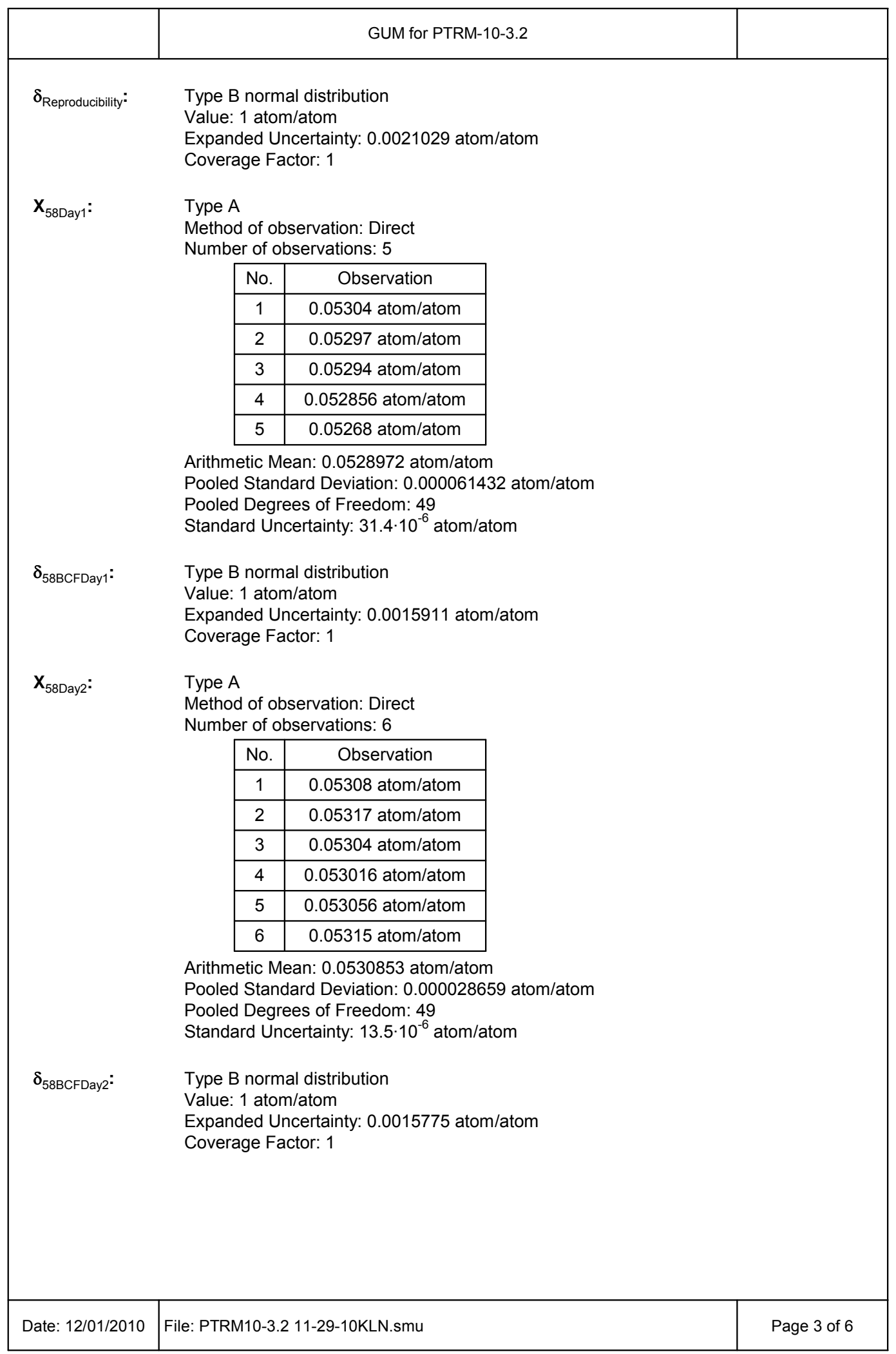


PNNL-20047

\begin{tabular}{|c|c|c|c|}
\hline & \multicolumn{2}{|r|}{ GUM for PTRM-10-3.2 } & \\
\hline \multirow[t]{8}{*}{$\mathrm{X}_{68 \text { Day } 1}:$} & \multicolumn{2}{|c|}{$\begin{array}{l}\text { Type A } \\
\text { Method of observation: Direct } \\
\text { Number of observations: } 5\end{array}$} & \\
\hline & No. & Observation & \\
\hline & 1 & 0.0001683 atom/atom & \\
\hline & 2 & 0.0001689 atom/atom & \\
\hline & 3 & 0.0001685 atom/atom & \\
\hline & 4 & 0.0001689 atom/atom & \\
\hline & 5 & 0.0001682 atom/atom & \\
\hline & \multicolumn{2}{|c|}{$\begin{array}{l}\text { Arithmetic Mean: } 168.5600 \cdot 10^{-6} \text { atom/atom } \\
\text { Pooled Standard Deviation: } 0.00000015870 \text { atom/atom } \\
\text { Pooled Degrees of Freedom: } 49 \\
\text { Standard Uncertainty: } 79.3 \cdot 10^{-9} \text { atom/atom }\end{array}$} & \\
\hline$\delta_{68 B C F D a y 1}:$ & \multicolumn{2}{|c|}{$\begin{array}{l}\text { Type B normal distribution } \\
\text { Value: } 1 \text { atom/atom } \\
\text { Expanded Uncertainty: } 0.0010607 \text { atom/atom } \\
\text { Coverage Factor: } 1\end{array}$} & \\
\hline \multirow[t]{9}{*}{$\mathbf{X}_{68 \text { Day2 }}$} & \multicolumn{2}{|c|}{$\begin{array}{l}\text { Type A } \\
\text { Method of observation: Direct } \\
\text { Number of observations: } 6\end{array}$} & \\
\hline & No. & Observation & \\
\hline & 1 & 0.0001660 atom/atom & \\
\hline & 2 & 0.0001662 atom/atom & \\
\hline & 3 & 0.0001692 atom/atom & \\
\hline & 4 & 0.0001673 atom/atom & \\
\hline & 5 & 0.0001682 atom/atom & \\
\hline & 6 & 0.0001683 atom/atom & \\
\hline & \multicolumn{2}{|c|}{$\begin{array}{l}\text { Arithmetic Mean: } 167.533 \cdot 10^{-6} \text { atom/atom } \\
\text { Pooled Standard Deviation: } 0.00000057448 \text { atom/atom } \\
\text { Pooled Degrees of Freedom: } 49 \\
\text { Standard Uncertainty: } 273 \cdot 10^{-9} \text { atom/atom }\end{array}$} & \\
\hline$\delta_{68 \mathrm{BCFDay2}}:$ & \multicolumn{2}{|c|}{$\begin{array}{l}\text { Type B normal distribution } \\
\text { Value: } 1 \text { atom/atom } \\
\text { Expanded Uncertainty: } 0.0010517 \text { atom/atom } \\
\text { Coverage Factor: } 1\end{array}$} & \\
\hline Date: $12 / 01 / 2010$ & \multicolumn{2}{|c|}{ File: PTRM10-3.2 11-29-10KLN.smu } & Page 4 of 6 \\
\hline
\end{tabular}

Generated with GUM Workbench Pro Version 2.4.1.332 


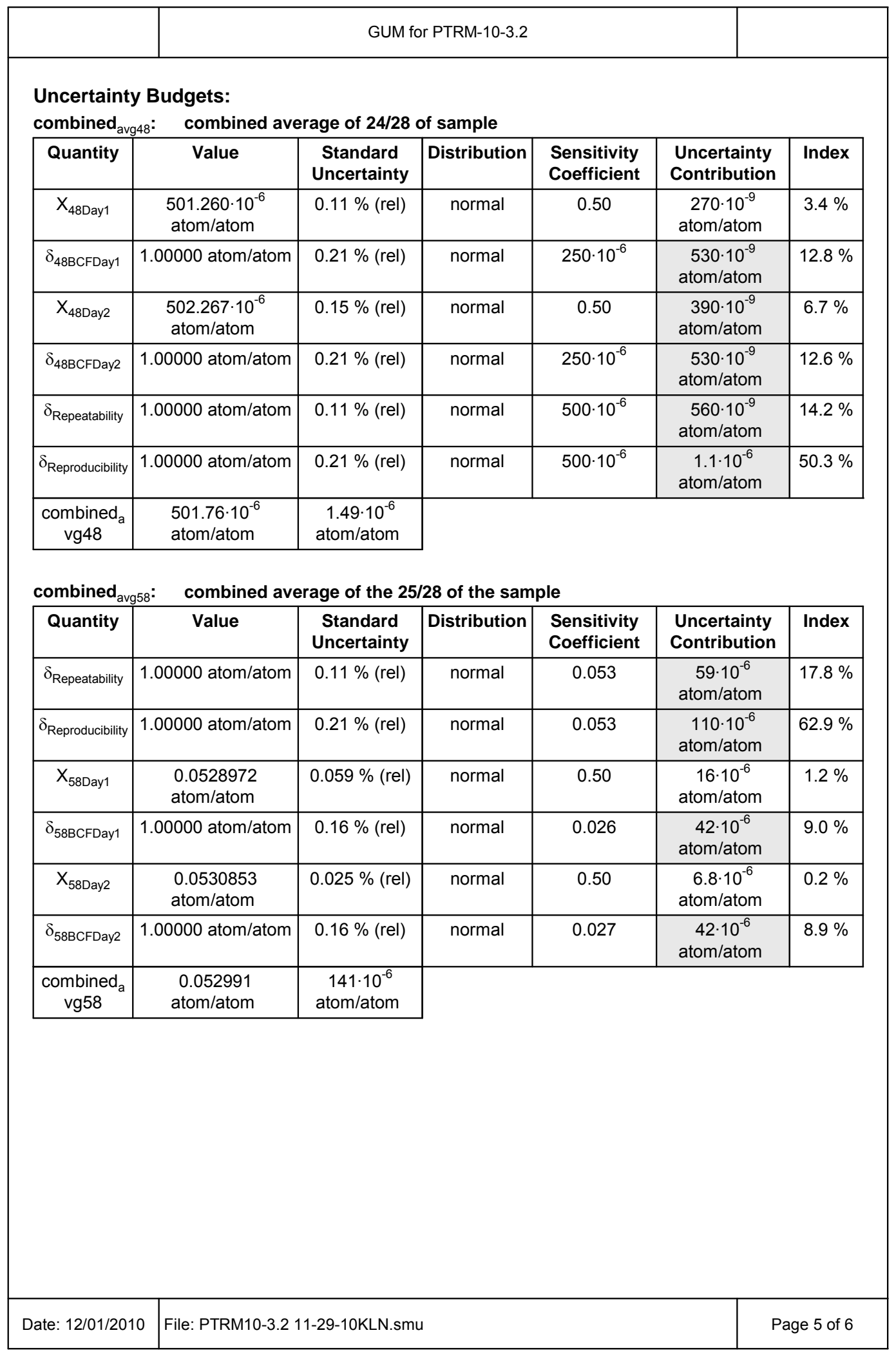




\begin{tabular}{|c|c|c|c|c|c|c|c|c|}
\hline & \multicolumn{6}{|c|}{ GUM for PTRM-10-3.2 } & & \\
\hline combined $_{\text {avg68: }}$ & \multicolumn{8}{|c|}{ combined average of the $26 / 28$ of the sample } \\
\hline Quantity & Value & \multicolumn{2}{|c|}{$\begin{array}{c}\text { Standard } \\
\text { Uncertainty }\end{array}$} & \multicolumn{2}{|c|}{ Distribution } & $\begin{array}{l}\text { Sensitivity } \\
\text { Coefficient }\end{array}$ & $\begin{array}{l}\text { Uncertainty } \\
\text { Contribution }\end{array}$ & Index \\
\hline$\delta_{\text {Repeatability }}$ & 1.00000 atom/atom & \multicolumn{2}{|c|}{$0.11 \%$ (rel) } & \multicolumn{2}{|c|}{ normal } & $170 \cdot 10^{-6}$ & $\begin{array}{c}190 \cdot 10^{-9} \\
\text { atom/atom }\end{array}$ & $18.0 \%$ \\
\hline \begin{tabular}{|l|l}
$\delta_{\text {Reproducibility }}$ & 1. \\
\end{tabular} & 1.00000 atom/atom & \multicolumn{2}{|c|}{$0.21 \%$ (rel) } & \multicolumn{2}{|c|}{ normal } & $170 \cdot 10^{-6}$ & $\begin{array}{c}350 \cdot 10^{-9} \\
\text { atom/atom }\end{array}$ & $63.7 \%$ \\
\hline $\mathrm{X}_{68 \mathrm{Day} 1}$ & $\begin{array}{c}168.5600 \cdot 10^{-6} \\
\text { atom/atom }\end{array}$ & \multicolumn{2}{|c|}{$0.047 \%$ (rel) } & \multicolumn{2}{|c|}{ normal } & 0.50 & $\begin{array}{c}40 \cdot 10^{-9} \\
\text { atom/atom }\end{array}$ & $0.8 \%$ \\
\hline$\delta_{68 \mathrm{BCFDay} 1}$ & 1.00000 atom/atom & \multicolumn{2}{|c|}{$0.11 \%($ rel) } & \multicolumn{2}{|c|}{ normal } & $84 \cdot 10^{-6}$ & $\begin{array}{c}89 \cdot 10^{-9} \\
\text { atom/atom }\end{array}$ & $4.1 \%$ \\
\hline $\mathrm{X}_{68 \text { Day2 }}$ & $\begin{array}{l}167.533 \cdot 10^{-6} \\
\text { atom/atom }\end{array}$ & \multicolumn{2}{|c|}{$0.16 \%$ (rel) } & \multicolumn{2}{|c|}{ normal } & 0.50 & $\begin{array}{c}140 \cdot 10^{-9} \\
\text { atom/atom }\end{array}$ & $9.5 \%$ \\
\hline$\delta_{68 B C F D a y 2}$ & 1.00000 atom/atom & \multicolumn{2}{|c|}{$0.11 \%(\mathrm{rel})$} & \multicolumn{2}{|c|}{ normal } & $84 \cdot 10^{-6}$ & $\begin{array}{c}88 \cdot 10^{-9} \\
\text { atom/atom }\end{array}$ & $4.0 \%$ \\
\hline $\begin{array}{c}\text { combined }_{a} \\
\text { vg68 }\end{array}$ & $\begin{array}{l}168.047 \cdot 10^{-6} \\
\text { atom/atom }\end{array}$ & $\begin{array}{r}443 \\
\text { atom }\end{array}$ & $\begin{array}{l}10^{-9} \\
\text { atom }\end{array}$ & & & & & \\
\hline \multicolumn{9}{|l|}{ Results: } \\
\hline Quantity & \multicolumn{2}{|l|}{ Value } & \multicolumn{2}{|c|}{$\begin{array}{l}\text { Expanded } \\
\text { Uncertainty }\end{array}$} & & $\begin{array}{l}\text { overage } \\
\text { factor }\end{array}$ & \multicolumn{2}{|l|}{ Coverage } \\
\hline combined $_{\text {avg48 }}$ & \multicolumn{2}{|c|}{$501.8 \cdot 10^{-6}$ atom/atom } & \multicolumn{2}{|c|}{$\begin{array}{c}3.0 \cdot 10^{-6} \\
\text { atom/atom }\end{array}$} & & 2.00 & \multicolumn{2}{|c|}{ 95\% (t-table $95.45 \%$ ) } \\
\hline combined $_{\text {avg58 }}$ & 0.05299 atom/a & tom & $\begin{array}{r}280 \\
\text { atom }\end{array}$ & $\begin{array}{l}10^{-6} \\
\text { latom }\end{array}$ & & 2.00 & 95\% (t-table 95.4 & $5 \%)$ \\
\hline combined $_{\text {avg68 }}$ & $168.05 \cdot 10^{-6}$ atom $/$ & latom & $\begin{array}{r}890 \\
\text { atom }\end{array}$ & $\begin{array}{l}\cdot 10^{-9} \\
\text { latom }\end{array}$ & & 2.00 & 95\% (t-table 95.4 & $5 \%)$ \\
\hline
\end{tabular}

